\title{
Robust Stability and Stability Radius for Variational Control Systems
}

\begin{abstract}
Bogdan Sasu
Department of Mathematics, Faculty of Mathematics and Computer Science, West University of Timişoara,
\end{abstract} Blvd. V. Pârvan 4, 300223 Timişoara, Romania

Correspondence should be addressed to Bogdan Sasu, bsasu@math.uvt.ro

Received 26 September 2007; Revised 15 January 2008; Accepted 27 February 2008

Recommended by Stephen Clark

We consider an integral variational control system on a Banach space $X$ and we study the connections between its uniform exponential stability and the $\left(I\left(\mathbb{R}_{+}, X\right), O\left(\mathbb{R}_{+}, X\right)\right)$ stability, where $I$ and $O$ are Banach function spaces. We identify the viable classes of input spaces and output spaces related to the exponential stability of systems and provide optimization techniques with respect to the input space. We analyze the robustness of exponential stability in the presence of structured perturbations. We deduce general estimations for the lower bound of the stability radius of a variational control system in terms of input-output operators acting on translation-invariant spaces. We apply the main results at the study of the exponential stability of nonautonomous systems and analyze in the nonautonomous case the robustness of this asymptotic property.

Copyright (C) 2008 Bogdan Sasu. This is an open access article distributed under the Creative Commons Attribution License, which permits unrestricted use, distribution, and reproduction in any medium, provided the original work is properly cited.

\section{Introduction}

In the past few years a significant progress was made in the asymptotic theory of dynamical systems and their applications in control theory (see [1-41]). It is well known that if $X$ is a Banach space, $\Theta$ is a compact metric space, $\sigma$ is a flow on $\Theta$, and $A: \Theta \rightarrow B(X)$ is a continuous mapping, then $\Phi(\theta, t)$, the solution operator of the linear differential equation

$$
\dot{x}(t)=A(\sigma(\theta, t)) x(t), \quad t \geq 0,
$$

is a cocycle and the pair $\pi=(\Phi, \sigma)$ is a linear skew-product flow. Often, (1.1) arises from the linearization of nonlinear equations (see $[9,31]$ and the references therein). Equation (1.1) is the starting point of our paper. We consider an integral model for the above type of systems, defined in terms of linear skew-product flows and we study the exponential stability of solutions. 
Input-output conditions in the study of the asymptotic properties of evolution equations have a long and impressive history that goes back to the work of Perron. In recent years, new ideas were developed in this theory, providing input-output theorems for stability, expansiveness, and dichotomy and also a number of applications in control theory (see $[2,7,9,11,13,15,19-27,29,30,32-41]$ and the references therein). The aim of our paper is to establish input-output conditions for uniform exponential stability of variational control systems, identifying the viable classes for input spaces and output spaces, as well. We provide a new approach based on the fundamental properties of Banach function spaces. Our attention is devoted to the relationship between the uniform exponential stability of a variational system and the stability of a pair of spaces which are translations invariant. We establish the connections between $\left(I\left(\mathbb{R}_{+}, X\right), O\left(\mathbb{R}_{+}, X\right)\right)$ stability and uniform exponential stability of a variational control system on a Banach space $X$, when the input space $I\left(\mathbb{R}_{+}, X\right)$ and the output space $O\left(\mathbb{R}_{+}, X\right)$ belong to specific classes of Banach function spaces. By examples we motivate our techniques and also discuss some optimization methods with respect to the input space.

In what follows, we consider a generalization of systems described by differential equations of the form

$$
\begin{gathered}
\dot{x}(t)=A(\sigma(\theta, t)) x(t)+B(\sigma(\theta, t)) u(t), \\
y(t)=C(\sigma(\theta, t)) x(t),
\end{gathered}
$$

where $\sigma$ is a flow on a locally compact metric space $\Theta, A(\theta)$ are unbounded operators on a Banach space $X$, and the operators $B(\theta) \in B(U, X), C(\theta) \in B(X, Y)$, where $U, Y$ are Banach spaces. Roughly speaking, the family $\{A(\theta)\}_{\theta \in \Theta}$ will be subjected to additive structured perturbations, so that the perturbed system is $\dot{x}(t)=[A(\sigma(\theta, t))+$ $B(\sigma(\theta, t)) \Delta(\sigma(\theta, t)) C(\sigma(\theta, t))] x(t)$, which may be interpreted as a system obtained by applying the feedback $u(t)=\Delta(\sigma(\theta, t)) y(t)$ to the time-varying system (1.2). The main question is how general may be the perturbation structure such that the property of exponential stability is preserved. We will answer this question by determining a general lower bound for the stability radius of such systems.

In the last decades, stability radius became a subject of large interest and various estimations for the lower bound of the stability radius of systems were obtained (see [11, 1720, 32, 33, 41]). This concept was introduced by Hinrichsen, Ilchmann, and Pritchard (see [18]) and led to a systematic study of the stability of linear infinite-dimensional systems under structured time-varying perturbations. The main idea was to estimate the size of the smallest disturbance operator under which the additively perturbed system loses exponential stability. A significant theorem which gives a lower bound for the stability radius of a system associated to a mild evolution operator in terms of the norm of the input-output operator of the system has been obtained by Hinrichsen and Pritchard (see [19, Theorem 3.2]). A distinct approach of this result was given by Clark et al. in [11], employing an evolution semigroup technique. The variational case was firstly treated in [32], where we obtained a lower bound for the stability radius in terms of the Perron operators associated with a linear skew-product semiflow. In [41], Wirth and Hinrichsen introduced a concept of stability radius for the case of discrete timevarying systems under structured perturbations of multi-output feedback type. In the spirit of this theory, we have obtained in [33] an estimation for the lower bound of the stability radius of a control system of difference equations on a Banach space $X$, under structured infinite multiperturbations, in terms of the norm of the input-output operator associated with a system 
on $l^{p}(\mathbb{N}, X)$ with $p \in[1, \infty]$. An interesting approach for systems in finite-dimensional spaces was presented by Jacob in [20]. There the author deduces a formula for the stability radius of time-varying systems with coefficients in $L_{\infty}^{\text {loc }}\left(0, \infty ; \mathbf{K}^{n \times n}\right)$, where $\mathbf{K} \in\{\mathbb{R}, \mathbf{C}\}$, in terms of the norms of a family of input-output operators.

In the fourth section of the present paper we propose a new approach, deducing a lower bound for the stability radius of variational systems in terms of input-output operators acting on rearrangement invariant Banach function spaces. Until now, the most common function spaces used for estimating the stability radius are the $L^{p}$-spaces with $p \in[1, \infty)$, which satisfy in particular the requirements of the classes introduced in this paper. Thus, our results provide a unified treatment in a large class of function spaces, extending the abovementioned contributions. As particular cases, we obtain a lower bound for the stability radius of a variational system in terms of $L^{p}$-spaces, with $p \in[1, \infty]$, and generalize the main result in [32].

In the last section, the central results of the paper are applied at the study of the uniform exponential stability of nonautonomous systems. We provide a complete analysis concerning the implications of the variational case for nonautonomous systems. We point out several interesting situations and deduce as particular cases the stability results due to Datko, Clark, Latushkin, Montgomery-Smith, Randolph, Neerven, Megan, and many others. Moreover, as an application we obtain a lower bound for the stability radius of nonautonomous systems in terms of the norm of the input-output operators acting on translations invariant spaces. Our results extend the existing contributions in the literature on this topic.

\section{Preliminary results on Banach function spaces}

Let $\mathcal{M}\left(\mathbb{R}_{+}, \mathbb{R}\right)$ be the linear space of all Lebesgue measurable functions $u: \mathbb{R}_{+} \rightarrow \mathbb{R}$, identifying the functions equal a.e.

Definition 2.1. A linear subspace $B$ of $\mathcal{M}\left(\mathbb{R}_{+}, \mathbb{R}\right)$ is called a normed function space if there is a mapping $|\cdot|_{B}: B \rightarrow \mathbb{R}_{+}$such that

(i) $|u|_{B}=0$ if and only if $u=0$ a.e.;

(ii) $|\alpha u|_{B}=|\alpha||u|_{B}$, for all $(\alpha, u) \in \mathbb{R} \times B$;

(iii) $|u+v|_{B} \leq|u|_{B}+|v|_{B}$, for all $u, v \in B$;

(iv) if $|u(t)| \leq|v(t)|$ a.e. $t \in \mathbb{R}_{+}$and $v \in B$, then $u \in B$ and $|u|_{B} \leq|v|_{B}$.

If $\left(B,|\cdot|_{B}\right)$ is complete, then $B$ is called a Banach function space.

Remark 2.2. (i) If $\left(B,|\cdot|_{B}\right)$ is a Banach function space and $u_{n} \rightarrow u$ in $B$, then there is a subsequence $\left(u_{k_{n}}\right)$ such that $u_{k_{n}} \rightarrow u$ a.e. (see, e.g., [28]).

(ii) If $\left(B,|\cdot|_{B}\right)$ is a Banach function space and $u \in B$, then $|u(\cdot)| \in B$.

Definition 2.3. A Banach function space $\left(B,|\cdot|_{B}\right)$ is said to be invariant to translations if for every $u: \mathbb{R}_{+} \rightarrow \mathbb{R}$ and every $t>0, u \in B$ if and only if the function

$$
u_{t}: \mathbb{R}_{+} \longrightarrow \mathbb{R}, \quad u_{t}(s)= \begin{cases}u(s-t), & s \geq t \\ 0, & s \in[0, t)\end{cases}
$$

belongs to $B$ and $\left|u_{t}\right|_{B}=|u|_{B}$. 
Let $C_{c}\left(\mathbb{R}_{+}, \mathbb{R}\right)$ be the linear space of all continuous functions $v: \mathbb{R}_{+} \rightarrow \mathbb{R}$ with compact support contained in $\mathbb{R}_{+}$and let $C_{0 c}\left(\mathbb{R}_{+}, \mathbb{R}\right):=\left\{v \in C_{c}\left(\mathbb{R}_{+}, \mathbb{R}\right): v(0)=0\right\}$. Let $L_{\text {loc }}^{1}\left(\mathbb{R}_{+}, \mathbb{R}\right)$ be the linear space of all locally integrable functions $u: \mathbb{R}_{+} \rightarrow \mathbb{R}$.

We denote by $\tau\left(\mathbb{R}_{+}\right)$the class of all Banach function spaces $B$ which are invariant to translations and with the following properties:

(i) $C_{c}\left(\mathbb{R}_{+}, \mathbb{R}\right) \subset B \subset L_{\text {loc }}^{1}\left(\mathbb{R}_{+}, \mathbb{R}\right)$;

(ii) if $B \backslash L^{1}\left(\mathbb{R}_{+}, \mathbb{R}\right) \neq \varnothing$, then there is a continuous function $\delta \in B \backslash L^{1}\left(\mathbb{R}_{+}, \mathbb{R}\right)$.

For every $A \subset \mathbb{R}_{+}$we denote by $\chi_{A}$ the characteristic function of the set $A$.

Remark 2.4. If $B \in \tau\left(\mathbb{R}_{+}\right)$, then $\chi_{[0, t)} \in B$, for all $t>0$.

Indeed, let $t>0$. If $\alpha: \mathbb{R}_{+} \rightarrow[0,1]$ is a continuous function with compact support such that $\alpha(s)=1$, for $s \in[0, t]$ and $\alpha(s)=0$, for $s \geq t+1$, then we have that $X[0, t)(s) \leq \alpha(s)$, for all $s \geq 0$. Since $\alpha \in B$, using (iv) from Definition 2.1, we deduce that $\chi_{[0, t)} \in B$.

Definition 2.5. If $B \in \tau\left(\mathbb{R}_{+}\right)$, then the function $F_{B}:(0, \infty) \rightarrow \mathbb{R}_{+}, F_{B}(t)=\left|X_{[0, t)}\right|_{B}$ is called the fundamental function of the space $B$.

Remark 2.6. The function $F_{B}$ is nondecreasing.

Example 2.7. Let $p \in[1, \infty)$. Then the space $L^{p}\left(\mathbb{R}_{+}, \mathbb{R}\right)=\left\{u \in \mathcal{M}\left(\mathbb{R}_{+}, \mathbb{R}\right): \int_{0}^{\infty}|u(\tau)|^{p} d \tau<\infty\right\}$ with respect to the norm $\|u\|_{p}=\left(\int_{0}^{\infty}|u(\tau)|^{p} d \tau\right)^{1 / p}$ is a Banach function space which belongs to $\tau\left(\mathbb{R}_{+}\right)$.

Example 2.8. Let $L^{\infty}\left(\mathbb{R}_{+}, \mathbb{R}\right)$ be the linear space of all essentially bounded functions $u \in$ $\mathcal{M}\left(\mathbb{R}_{+}, \mathbb{R}\right)$. With respect to the norm $\|u\|_{\infty}:=\operatorname{ess~sup}_{t \geq 0}|u(t)|, L^{\infty}\left(\mathbb{R}_{+}, \mathbb{R}\right)$ is a Banach function space which belongs to $\tau\left(\mathbb{R}_{+}\right)$.

Example 2.9 (Orlicz spaces). Let $\varphi: \mathbb{R}_{+} \rightarrow \mathbb{R}_{+}$be a nondecreasing left-continuous function, which is not identically zero on $(0, \infty)$. The Young function associated with $\varphi$ is defined by $Y_{\varphi}(t)=$ $\int_{0}^{t} \varphi(s) d s$. For every $u \in \mathcal{M}\left(\mathbb{R}_{+}, \mathbb{R}\right)$ we define $M_{\varphi}(u):=\int_{0}^{\infty} Y_{\varphi}(|u(s)|) d s$. The set $O_{\varphi}$ of all $u \in$ $\mathcal{M}\left(\mathbb{R}_{+}, \mathbb{R}\right)$ with the property that there is $k>0$ such that $M_{\varphi}(k u)<\infty$ is a linear space. With respect to the norm $|u|_{\varphi}:=\inf \left\{k>0: M_{\varphi}(u / k) \leq 1\right\}, O_{\varphi}$ is a Banach space called the Orlicz space associated with $\varphi$.

Remark 2.10. It is easy to verify that the Orlicz spaces introduced in Example 2.9 belong to $\tau\left(\mathbb{R}_{+}\right)$.

Remark 2.11. The spaces $L^{p}\left(\mathbb{R}_{+}, \mathbb{R}\right), p \in[1, \infty]$ are Orlicz spaces, which may be obtained for $\varphi(t)=p t^{p-1}$, if $p \in[1, \infty)$ and for

$$
\varphi(t)=\left\{\begin{array}{ll}
0, & t \in[0,1] \\
1, & t>1
\end{array} \text { if } p=\infty\right.
$$

Lemma 2.12. Let $B \in \tau\left(\mathbb{R}_{+}\right)$and $v>0$. Then the function $e_{v}: \mathbb{R}_{+} \rightarrow \mathbb{R}_{+}, e_{v}(t)=e^{-v t}$, belongs to $B$. 
Proof. We have that

$$
e_{v}(t)=\sum_{n=0}^{\infty} e^{-v t} X_{[n, n+1)}(t) \leq \sum_{n=0}^{\infty} e^{-v n} X_{[n, n+1)}(t), \quad \forall t \geq 0 .
$$

This shows that $e_{v} \in B$ and $\left|e_{v}\right|_{B} \leq\left|X_{[0,1)}\right|_{B} /\left(1-e^{-v}\right)$.

Definition 2.13. Let $u, v \in \mathcal{M}\left(\mathbb{R}_{+}, \mathbb{R}\right)$. Say that $u$ and $v$ are equimeasurable if for every $t>0$ the sets $\left\{s \in \mathbb{R}_{+}:|u(s)|>t\right\}$ and $\left\{s \in \mathbb{R}_{+}:|v(s)|>t\right\}$ have the same measure.

Definition 2.14. A Banach function space $\left(B,|\cdot|_{B}\right)$ is rearrangement invariant if for every equimeasurable functions $u, v: \mathbb{R}_{+} \rightarrow \mathbb{R}_{+}$with $u \in B$, one has $v \in B$ and $|u|_{B}=|v|_{B}$.

Remark 2.15. The Orlicz spaces are rearrangement invariant (see [4, Theorem 8.9]).

We denote by $\mathcal{R}\left(\mathbb{R}_{+}\right)$the class of all Banach function spaces $B \in \tau\left(\mathbb{R}_{+}\right)$which are rearrangement invariant.

Remark 2.16. If $B \in \mathcal{R}\left(\mathbb{R}_{+}\right)$, then $B$ is an interpolation space between $L^{1}\left(\mathbb{R}_{+}, \mathbb{R}\right)$ and $L^{\infty}\left(\mathbb{R}_{+}, \mathbb{R}\right)$ (see [4, Theorem 2.2, page 106]).

Lemma 2.17. Let $B \in \tau\left(\mathbb{R}_{+}\right)$and $v>0$. Then for every $u \in B$ the function

$$
\psi_{u}: \mathbb{R}_{+} \longrightarrow \mathbb{R}, \quad \psi_{u}(t)=\int_{0}^{t} e^{-v(t-s)} u(s) d s
$$

belongs to $B$. Moreover, there is $\lambda_{B}>0$ such that $\left|\psi_{u}\right|_{B} \leq \lambda_{B}|u|_{B}$, for all $u \in B$.

Proof. It is easy to see that

$$
D_{v}: L^{\infty}\left(\mathbb{R}_{+}, \mathbb{R}\right) \longrightarrow L^{\infty}\left(\mathbb{R}_{+}, \mathbb{R}\right), \quad\left(D_{v}(u)\right)(t)=\int_{0}^{t} e^{-v(t-s)} u(s) d s
$$

is a correctly defined bounded linear operator. Moreover, the restriction $D_{v \mid}$ : $L^{1}\left(\mathbb{R}_{+}, \mathbb{R}\right) \rightarrow L^{1}\left(\mathbb{R}_{+}, \mathbb{R}\right)$ is correctly defined and is a bounded linear operator. Then from Remark 2.16 it follows that $D_{\left.v\right|_{B}}: B \rightarrow B$ is a correctly defined and bounded linear operator. Taking $\lambda_{B}=\left\|D_{\left.v\right|_{B}}\right\|$, the proof is complete.

We denote by $Q\left(\mathbb{R}_{+}\right)$the class of all Banach function spaces $B \in \tau\left(\mathbb{R}_{+}\right)$with the property that $\sup _{t>0} F_{B}(t)=\infty$.

Remark 2.18. If $\varphi(t) \in(0, \infty)$, for all $t>0$, then the Orlicz space $O_{\varphi} \in \mathcal{Q}\left(\mathbb{R}_{+}\right)$(see, e.g., [24, Proposition 2.1]).

Let $C_{0}\left(\mathbb{R}_{+}, \mathbb{R}\right)$ be the space of all continuous functions $u: \mathbb{R}_{+} \rightarrow \mathbb{R}$ with $\lim _{t \rightarrow \infty} u(t)=0$.

Lemma 2.19. If $B \in \tau\left(\mathbb{R}_{+}\right) \backslash Q\left(\mathbb{R}_{+}\right)$, then $C_{0}\left(\mathbb{R}_{+}, \mathbb{R}\right) \subset B$.

Proof. Let $u \in C_{0}\left(\mathbb{R}_{+}, \mathbb{R}\right)$. Then there is an unbounded increasing sequence $\left(t_{n}\right) \subset(0, \infty)$ such that $|u(t)| \leq 1 /(n+1)$, for all $t \geq t_{n}$ and $n \in \mathbb{N}$. We set $u_{n}=u \chi_{\left[0, t_{n}\right)}$. According to our hypothesis, $M:=\sup _{t>0} F_{B}(t)<\infty$. Then, we observe that

$$
\left|u_{n+p}-u_{n}\right|_{B} \leq \frac{1}{n+1}\left|\chi_{\left[t_{n}, t_{n+p}\right)}\right|_{B} \leq \frac{M}{n+1}, \quad \forall p \in \mathbb{N}^{*}, \forall n \in \mathbb{N} .
$$


Hence, the sequence $\left(u_{n}\right)$ is fundamental in $B$, so there is $v \in B$ such that $u_{n} \rightarrow v$ in $B$. From Remark 2.2 there exists a subsequence $\left(u_{k_{n}}\right)$ such that $u_{k_{n}} \rightarrow v$ a.e. This implies that $u=v$ a.e., so $u=v$ in $B$. It follows that $u \in B$ and the proof is complete.

We denote by $\mathcal{L}\left(\mathbb{R}_{+}\right)$the class of all Banach function spaces $B \in \tau\left(\mathbb{R}_{+}\right)$with the property that $B \backslash L^{1}\left(\mathbb{R}_{+}, \mathbb{R}\right) \neq \varnothing$.

Remark 2.20. Using Remark 2.2(ii) we have that if $B \in \mathcal{L}\left(\mathbb{R}_{+}\right)$, then there is a continuous function $\delta: \mathbb{R}_{+} \rightarrow \mathbb{R}_{+}$with $\delta \in B \backslash L^{1}\left(\mathbb{R}_{+}, \mathbb{R}\right)$.

Lemma 2.21. If $B \in \tau\left(\mathbb{R}_{+}\right)$, then $B \in \mathcal{Q}\left(\mathbb{R}_{+}\right)$or $B \in \mathcal{L}\left(\mathbb{R}_{+}\right)$.

Proof. Suppose by contrary that there exists $B \in \tau\left(\mathbb{R}_{+}\right)$such that $B \notin Q\left(\mathbb{R}_{+}\right)$and $B \notin \mathcal{L}\left(\mathbb{R}_{+}\right)$. Then $M:=\sup _{t>0} F_{B}(t)<\infty$ and $B \subset L^{1}\left(\mathbb{R}_{+}, \mathbb{R}\right)$. It follows that there is $c>0$ such that $\|u\|_{1} \leq c|u|_{B}$, for all $u \in B$. In particular, for $u=\chi_{[0, t)}$ we deduce that $t \leq c\left|\chi_{[0, t)}\right|_{B}=c F_{B}(t) \leq c M$, for all $t>0$, which is absurd.

Let $(X,\|\cdot\|)$ be a real or complex Banach space. We denote by $C_{c}\left(\mathbb{R}_{+}, X\right)$ the linear space of all continuous functions $v: \mathbb{R}_{+} \rightarrow X$ with compact support, let $C_{0 c}=\left\{v \in C_{c}\left(\mathbb{R}_{+}, X\right): v(0)=0\right\}$ and let $L_{\text {loc }}^{1}\left(\mathbb{R}_{+}, X\right)$ be the linear space of all locally integrable functions $u: \mathbb{R}_{+} \rightarrow X$.

For every $B \in \tau\left(\mathbb{R}_{+}\right)$we denote by $B\left(\mathbb{R}_{+}, X\right)$ the linear space of all Bochner measurable functions $u: \mathbb{R}_{+} \rightarrow X$ with the property that the mapping $N_{u}: \mathbb{R}_{+} \rightarrow \mathbb{R}_{+}, N_{u}(t)=\|u(t)\|$ lies in $B$. Endowed with the norm $\|u\|_{B\left(\mathbb{R}_{+}, X\right)}:=\left|N_{u}\right|_{B}, B\left(\mathbb{R}_{+}, X\right)$ is a Banach space.

\section{Stability of variational integral control systems}

In the stability theory of evolution operators in Banach spaces, an evolution family $\boldsymbol{U}=$ $\{U(t, s)\}_{t \geq s \geq 0}$ is said to be $\left(E\left(\mathbb{R}_{+}, X\right), F\left(\mathbb{R}_{+}, X\right)\right)$ stable if for every $f \in E\left(\mathbb{R}_{+}, X\right)$ the mapping $P_{f}$ belongs to $F\left(\mathbb{R}_{+}, X\right)$ where

$$
P_{f}(t)=\int_{0}^{t} U(t, s) f(s) d s
$$

The classical input-output results concerning exponential stability of evolution families in Banach spaces may be stated as follows.

Let $\mathcal{U}=\{U(t, s)\}_{t \geq s \geq 0}$ be an evolution operator on a Banach space $X$ and $p, q \in[1, \infty)$ with $p \leq q$. Then the following assertions are equivalent:

(i) $\mathcal{U}$ is uniformly exponentially stable;

(ii) $\mathcal{U}$ is $\left(C_{0}\left(\mathbb{R}_{+}, X\right), C_{0}\left(\mathbb{R}_{+}, X\right)\right)$ stable;

(iii) $\mathcal{U}$ is $\left(C_{0}\left(\mathbb{R}_{+}, X\right), C_{b}\left(\mathbb{R}_{+}, X\right)\right)$ stable;

(iv) $\mathcal{U}$ is $\left(L^{p}\left(\mathbb{R}_{+}, X\right), L^{q}\left(\mathbb{R}_{+}, X\right)\right)$ stable.

The implication (iv) $\Rightarrow$ (i) was obtained by Datko in 1973 (see [16]), using one of his stability results contained in the same paper. In the last few years, there were pointed out new and interesting methods of proving the above theorem. The equivalences (i) $\Leftrightarrow$ (ii) $\Leftrightarrow$ (iv) were proved by Neerven for the special case of $C_{0}$ semigroups of linear operators (see [30]) and $p=q$. In [11], Clark et al. have provided an inedit technique of proving the equivalences 
(i) $\Leftrightarrow($ ii) $\Leftrightarrow$ (iv) for the case $p=q$. There the authors established important connections between the asymptotic properties of evolution families and those of the associated evolution semigroups with a large number of applications in control theory. Roughly speaking the authors described the behavior of a nonautonomous system in terms of the properties of an associated autonomous system. Therefore, in their approach the input space must coincide with the output space.

The equivalence (i) $\Leftrightarrow$ (ii) $\Leftrightarrow$ (iii) has been also proved by van Minh et al. (see [29]), employing an evolution semigroup technique. The equivalence (i) $\Leftrightarrow$ (ii) was also treated by Buşe in [7]. The case $p \neq q$ was completely treated in [23], where several new stability results of Perron type were established for exponential stability of evolution families, generalizing the above equivalences and obtaining that uniform exponential stability of an evolution family can be expressed using boundedly locally dense subsets of $C_{b}\left(\mathbb{R}_{+}, X\right)$ and $L^{p}\left(\mathbb{R}_{+}, X\right)$, respectively. The main results in [23] were extended in [25] for the case of linear skew-product flows, where the input-output operator was replaced with a family of operators acting between the function spaces of the admissible pair. The stability results obtained in [25] generalized some theorems from $[7,16,23,29,30]$. Moreover, the stability theorems in [25] led to several interesting consequences in control theory (see $[26,32]$ ).

In what follows, the input-output techniques in the stability theory of variational equations will be treated from the perspective of Banach function spaces arising from the interpolation theory. In this section, our main purpose is to give a complete and unified study of the exponential stability of variational systems via input-output methods, providing the "structure" of the classes of input and output spaces, respectively.

Let $X$ be a Banach space, let $(\Theta, d)$ be a metric space, and let $\varepsilon=X \times \Theta$. We denote by $B(X)$ the Banach algebra of all bounded linear operators on $X$. The norm on $X$ and on $B(X)$ will be denoted by $\|\cdot\|$.

Definition 3.1. Let $J \in\left\{\mathbb{R}_{+}, \mathbb{R}\right\}$. A continuous mapping $\sigma: \Theta \times J \rightarrow \Theta$ is called a flow on $\Theta$ if $\sigma(\theta, 0)=\theta$ and $\sigma(\theta, s+t)=\sigma(\sigma(\theta, s), t)$, for all $(\theta, s, t) \in \Theta \times J^{2}$.

Definition 3.2. A pair $\pi=(\Phi, \sigma)$ is called a linear skew-product flow on $\varepsilon=X \times \Theta$ if $\sigma$ is a flow on $\Theta$ and $\Phi: \Theta \times \mathbb{R}_{+} \rightarrow \mathbb{B}(X)$ satisfies the following conditions:

(i) $\Phi(\theta, 0)=I_{d}$, the identity operator on $X$, for all $\theta \in \Theta$;

(ii) $\Phi(\theta, t+s)=\Phi(\sigma(\theta, t), s) \Phi(\theta, t)$, for all $(\theta, t, s) \in \Theta \times \mathbb{R}_{+}^{2}$ (the cocycle identity);

(iii) $(\theta, t) \mapsto \Phi(\theta, t) x$ is continuous, for every $x \in X$;

(iv) there are $M \geq 1$ and $\omega>0$ such that $\|\Phi(\theta, t)\| \leq M e^{\omega t}$, for all $(\theta, t) \in \Theta \times \mathbb{R}_{+}$.

The mapping $\Phi$ given by Definition 3.2 is called the cocycle associated to the linear skewproduct flow $\pi=(\Phi, \sigma)$.

Let $\pi=(\Phi, \sigma)$ be a linear skew-product flow on $\varepsilon=X \times \Theta$. In what follows, we consider the variational integral control system

$$
x_{\theta}\left(t ; x_{0}, u\right)=\Phi(\theta, t) x_{0}+\int_{0}^{t} \Phi(\sigma(\theta, s), t-s) u(s) d s, \quad t \geq 0, \theta \in \Theta
$$

with $u \in L_{\text {loc }}^{1}\left(\mathbb{R}_{+}, X\right)$ and $x_{0} \in X$. 
Definition 3.3. The system $(S)$ is said to be

(i) uniformly stable if there is $L>0$ such that

$$
\left\|x_{\theta}\left(t ; x_{0}, 0\right)\right\| \leq L\left\|x_{0}\right\|, \quad \forall(\theta, t) \in \Theta \times \mathbb{R}_{+}, \forall x_{0} \in X
$$

(ii) uniformly exponentially stable if there are $K, v>0$ such that

$$
\left\|x_{\theta}\left(t ; x_{0}, 0\right)\right\| \leq K e^{-v t}\left\|x_{0}\right\|, \quad \forall(\theta, t) \in \Theta \times \mathbb{R}_{+}, \forall x_{0} \in X .
$$

Remark 3.4. (i) The system (S) is uniformly stable if and only if there is $L>0$ such that $\|\Phi(\theta, t)\| \leq L$, for all $(\theta, t) \in \Theta \times \mathbb{R}_{+}$.

(ii) The system $(S)$ is uniformly exponentially stable if and only if there are $K, v>0$ such that $\|\Phi(\theta, t)\| \leq K e^{-v t}$, for all $(\theta, t) \in \Theta \times \mathbb{R}_{+}$.

Definition 3.5. Let $I, O$ be two Banach function spaces with $I, O \in \tau\left(\mathbb{R}_{+}\right)$. The system $(S)$ is said to be $\left(I\left(\mathbb{R}_{+}, X\right), O\left(\mathbb{R}_{+}, X\right)\right)$ stable if the following assertions hold:

(i) for every $u \in C_{0 c}\left(\mathbb{R}_{+}, X\right)$ and every $\theta \in \Theta$ the solution $x_{\theta}(\cdot ; 0, u) \in O\left(\mathbb{R}_{+}, X\right)$;

(ii) there is $\lambda>0$ such that $\left\|x_{\theta}(\cdot ; 0, u)\right\|_{O\left(\mathbb{R}_{+}, X\right)} \leq \lambda\|u\|_{I\left(\mathbb{R}_{+}, X\right)}$, for all $(u, \theta) \in C_{0 c}\left(\mathbb{R}_{+}, X\right) \times \Theta$.

$I\left(\mathbb{R}_{+}, X\right)$ is called the input space and $O\left(\mathbb{R}_{+}, X\right)$ is called the output space.

We begin with a sufficient condition for uniform stability.

Theorem 3.6. Let $I, O \in \tau\left(\mathbb{R}_{+}\right)$. If the system $(S)$ is $\left(I\left(\mathbb{R}_{+}, X\right), O\left(\mathbb{R}_{+}, X\right)\right)$ stable, then the system $(S)$ is uniformly stable.

Proof. Let $\alpha: \mathbb{R}_{+} \rightarrow[0,2]$ be a continuous function with supp $\alpha \subset(0,1)$ and $\int_{0}^{1} \alpha(\tau) d \tau=1$.

Let $M, \omega \in(0, \infty)$ be given by Definition 3.2 and let $\lambda>0$ be given by Definition 3.5.

Let $(x, \theta) \in X \times \Theta$. We consider the function $u: \mathbb{R}_{+} \rightarrow X, u(t)=\alpha(t) \Phi(\theta, t) x$. We have that $u \in C_{0 c}\left(\mathbb{R}_{+}, X\right)$ and $\|u(t)\|=\alpha(t)\|\Phi(\theta, t) x\| \leq M e^{\omega}\|x\| \alpha(t)$, for all $t \geq 0$. This implies that

$$
\|u\|_{I\left(\mathbb{R}_{+}, X\right)} \leq M e^{\omega}\|x\||\alpha|_{I} .
$$

From hypothesis we have that $x_{\theta}(\cdot ; 0, u) \in O\left(\mathbb{R}_{+}, X\right)$ and

$$
\left\|x_{\theta}(\cdot ; 0, u)\right\|_{O\left(\mathbb{R}_{+}, X\right)} \leq \lambda\|u\|_{I\left(\mathbb{R}_{+}, X\right)} .
$$

We observe that

$$
\begin{aligned}
x_{\theta}(t ; 0, u) & =\int_{0}^{t} \Phi(\sigma(\theta, s), t-s) u(s) d s \\
& =\left(\int_{0}^{t} \alpha(s) d s\right) \Phi(\theta, t) x=\Phi(\theta, t) x, \quad \forall t \geq 1 .
\end{aligned}
$$

Then, for $t \geq 2$ we deduce that

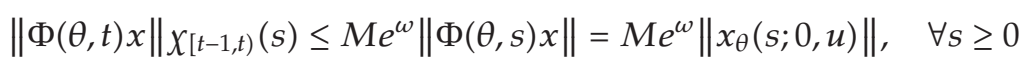


which implies that

$$
\|\Phi(\theta, t) x\|\left|x_{[t-1, t)}\right|_{O} \leq M e^{\omega}\left\|x_{\theta}(\cdot ; 0, u)\right\|_{O\left(\mathbb{R}_{+}, X\right)} .
$$

Since $O$ is invariant to translations we have that $\left|\chi_{[t-1, t)}\right|_{O}=\left|\chi_{[0,1)}\right|_{O}=F_{O}(1)$. Then, from relations (3.4)-(3.8) we obtain that

$$
\|\Phi(\theta, t) x\| \leq \frac{\lambda M e^{\omega}}{F_{O}(1)}\|u\|_{I\left(\mathbb{R}_{+}, X\right)} \leq \frac{\lambda M^{2} e^{2 \omega}|\alpha|_{I}}{F_{O}(1)}\|x\|, \quad \forall t \geq 2 .
$$

For $t \in[0,2]$ we have that $\|\Phi(\theta, t)\| \leq M e^{2 \omega}$. Then, for $L=\max \left\{M e^{2 \omega},\left(\lambda M^{2} e^{2 \omega}|\alpha|_{I}\right) / F_{O}(1)\right\}$ we deduce that $\|\Phi(\theta, t) x\| \leq L\|x\|$, for all $t \geq 0$. Taking into account that $L$ does not depend on $\theta$ or $x$, it follows that $\|\Phi(\theta, t) x\| \leq L\|x\|$, for all $(\theta, t) \in \Theta \times \mathbb{R}_{+}$and all $x \in X$ and the proof is complete.

The first main result of this section is the following.

Theorem 3.7. Let $I, O \in \tau\left(\mathbb{R}_{+}\right)$. If $I \in \mathcal{L}\left(\mathbb{R}_{+}\right)$and the system $(S)$ is $\left(I\left(\mathbb{R}_{+}, X\right), O\left(\mathbb{R}_{+}, X\right)\right)$ stable, then the system $(S)$ is uniformly exponentially stable.

Proof. Let $\lambda>0$ be given by Definition 3.5. From Theorem 3.6 we have that there is $L>0$ such that $\|\Phi(\theta, t)\| \leq L$, for all $(\theta, t) \in \Theta \times \mathbb{R}_{+}$.

Since $I \in \mathcal{L}\left(\mathbb{R}_{+}\right)$from Remark 2.20 it follows that there is a continuous function $\delta$ : $\mathbb{R}_{+} \rightarrow \mathbb{R}_{+}$such that $\delta \in I \backslash L^{1}\left(\mathbb{R}_{+}, \mathbb{R}\right)$. Let $h>0$ be such that

$$
\int_{0}^{h} \delta(s) d s \geq 2 e \frac{\lambda L^{2}|\delta|_{I}}{F_{O}(1)}
$$

For every $n \in \mathbb{N}^{*}$ let $\alpha_{n}: \mathbb{R}_{+} \rightarrow[0,1]$ be a continuous function with $\alpha_{n}(t)=1$, for $t \in$ $[1 / n, h], \alpha_{n}(0)=0$, and $\alpha_{n}(t)=0$, for $t \geq h+1$. Since

$$
\int_{0}^{h} \alpha_{n}(s) \delta(s) d s \longrightarrow \int_{0}^{h} \delta(s) d s, \quad \text { as } n \longrightarrow \infty
$$

there is $j \in \mathbb{N}^{*}$ such that

$$
a:=\int_{0}^{h} \alpha_{j}(s) \delta(s) d s \geq \frac{1}{2} \int_{0}^{h} \delta(s) d s \geq e \frac{\lambda L^{2}|\delta|_{I}}{F_{O}(1)} .
$$

Let $(x, \theta) \in \mathcal{E}=X \times \Theta$. We consider the function

$$
u: \mathbb{R}_{+} \longrightarrow X, \quad u(t)=\alpha_{j}(t) \delta(t) \Phi(\theta, t) x .
$$

We have that $u \in C_{0 c}\left(\mathbb{R}_{+}, X\right)$, so $u \in I\left(\mathbb{R}_{+}, X\right)$. In addition, $\|u(t)\| \leq L\|x\| \delta(t)$, for all $t \geq 0$. This implies that $\|u\|_{I\left(\mathbb{R}_{+}, X\right)} \leq L\|x\||\delta|_{I}$. We observe that

$$
\begin{aligned}
\left\|x_{\theta}(t ; 0, u)\right\| & =\left\|\int_{0}^{t} \Phi(\sigma(\theta, s), t-s) u(s) d s\right\|=\left(\int_{0}^{t} \alpha_{j}(s) \delta(s) d s\right)\|\Phi(\theta, t) x\| \\
& \geq a\|\Phi(\theta, t) x\|, \quad \forall t \geq h .
\end{aligned}
$$


Then, we have that

$$
\|\Phi(\theta, h+1) x\| \leq L\|\Phi(\theta, t) x\| \leq \frac{L}{a}\left\|x_{\theta}(t ; 0, u)\right\|, \quad \forall t \in[h, h+1)
$$

which implies that

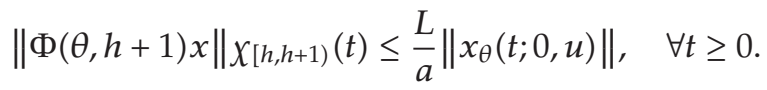

Since $O$ is invariant to translations we have that $\left|\chi_{[h, h+1)}\right|_{O}=\left|\chi_{[0,1)}\right|_{O}=F_{O}(1)$. Then from (3.16) we deduce that

$$
\|\Phi(\theta, h+1) x\| F_{O}(1) \leq \frac{L}{a}\left\|x_{\theta}(\cdot ; 0, u)\right\|_{O\left(\mathbb{R}_{+}, X\right)} .
$$

According to our hypothesis

$$
\left\|x_{\theta}(\cdot ; 0, u)\right\|_{O\left(\mathbb{R}_{+}, X\right)} \leq \lambda\|u\|_{I\left(\mathbb{R}_{+}, X\right)} .
$$

Using relations (3.17) and (3.18) it follows that

$$
a\|\Phi(\theta, h+1) x\| \leq \frac{\lambda L}{F_{O}(1)}\|u\|_{I\left(\mathbb{R}_{+}, X\right)} \leq \frac{\lambda L^{2}|\delta|_{I}}{F_{O}(1)}\|x\| .
$$

We set $r=h+1$. From (3.19) and (3.12) we obtain that $\|\Phi(\theta, r) x\| \leq(1 / e)\|x\|$. Since $r$ does not depend on $\theta$ or $x$ we deduce that $\|\Phi(\theta, r) x\| \leq(1 / e)\|x\|$, for all $(x, \theta) \in \mathcal{E}$.

Let $v=1 / r$ and $K=L e$. Let $t \geq 0$. Then there is $n \in \mathbb{N}$ and $s \in[0, r)$ such that $t=n r+s$. It follows that $\|\Phi(\theta, t)\| \leq L\|\Phi(\theta, n r)\| \leq L e^{-n} \leq K e^{-v t}$. This implies that the system $(S)$ is uniformly exponentially stable.

The second main result of this section is the following.

Theorem 3.8. Let $I, O \in \tau\left(\mathbb{R}_{+}\right)$. If $O \in \mathcal{Q}\left(\mathbb{R}_{+}\right)$and the system $(S)$ is $\left(I\left(\mathbb{R}_{+}, X\right), O\left(\mathbb{R}_{+}, X\right)\right)$ stable, then $(S)$ is uniformly exponentially stable.

Proof. Let $\lambda>0$ be given by Definition 3.5 and let $L>0$ be given by Theorem 3.6. Let $h>0$ be such that

$$
F_{O}(h) \geq 2 e \lambda L^{2} F_{I}(1)
$$

Let $\alpha: \mathbb{R}_{+} \rightarrow[0,2]$ be a continuous function with supp $\alpha \subset(0,1)$ and $\int_{0}^{1} \alpha(\tau) d \tau=1$.

Let $(x, \theta) \in \mathcal{\varepsilon}=X \times \Theta$. We consider the function

$$
u: \mathbb{R}_{+} \longrightarrow X, \quad u(t)=\alpha(t) \Phi(\theta, t) x
$$

Since $u \in C_{0 c}\left(\mathbb{R}_{+}, X\right)$ we have that $u \in I\left(\mathbb{R}_{+}, X\right)$. Moreover, $\|u(t)\| \leq L\|x\| \alpha(t) \leq 2 L\|x\|_{X[0,1)}(t)$, for all $t \geq 0$, which implies that

$$
\|u\|_{I\left(\mathbb{R}_{+}, X\right)} \leq 2 L\|x\| F_{I}(1) .
$$


We observe that $x_{\theta}(t, 0, u)=\Phi(\theta, t) x$, for all $t \geq 1$. Then, we have that

$$
\|\Phi(\theta, h+1) x\|_{[[1, h+1)}(t) \leq L\|\Phi(\theta, t) x\| \chi_{[1, h+1)}(t) \leq L\left\|x_{\theta}(t ; 0, u)\right\|, \quad \forall t \geq 0
$$

Since $O \in \tau\left(\mathbb{R}_{+}\right)$we have that $\left|\chi_{[1, h+1)}\right|_{O}=\left|X_{[0, h)}\right|_{O}=F_{O}(h)$. Then from (3.23) and (3.22) we deduce that

$$
\|\Phi(\theta, h+1) x\| F_{O}(h) \leq L\left\|x_{\theta}(\cdot ; 0, u)\right\|_{O\left(\mathbb{R}_{+}, X\right)} \leq \lambda L\|u\|_{I\left(\mathbb{R}_{+}, X\right)} \leq 2 \lambda L^{2} F_{I}(1)\|x\| .
$$

From relations (3.20) and (3.24) it follows that $\|\Phi(\theta, h+1) x\| \leq(1 / e)\|x\|$. Taking into account that $h$ does not depend on $\theta$ or $x$ we obtain that $\|\Phi(\theta, h+1) x\| \leq(1 / e)\|x\|$, for all $(x, \theta) \in \mathcal{E}=$ $X \times \Theta$. Using similar arguments as in Theorem 3.7 we obtain that the system $(S)$ is uniformly exponentially stable.

The central result of this section is the following.

Theorem 3.9. Let $I, O \in \mathcal{C}\left(\mathbb{R}_{+}\right)$be such that $I \in \mathcal{L}\left(\mathbb{R}_{+}\right)$or $O \in \mathcal{Q}\left(\mathbb{R}_{+}\right)$. Then, the following assertions hold:

(i) if the system $(S)$ is $\left(I\left(\mathbb{R}_{+}, X\right), O\left(\mathbb{R}_{+}, X\right)\right)$ stable, then $(S)$ is uniformly exponentially stable;

(ii) if $I \subset O$ and one of the spaces $I, O$ belongs to the class $R\left(\mathbb{R}_{+}\right)$, then the system $(S)$ is uniformly exponentially stable if and only if the system $(S)$ is $\left(I\left(\mathbb{R}_{+}, X\right), O\left(\mathbb{R}_{+}, X\right)\right)$ stable.

Proof. (i) This follows from Theorems 3.7 and 3.8.

(ii) Necessity. Let $K, v>0$ be such that $\|\Phi(\theta, t) x\| \leq K e^{-v t}\|x\|$, for all $(x, \theta) \in \mathcal{\varepsilon}$ and all $t \geq 0$. Since $I \subset O$, there is $\gamma>0$ such that

$$
|w|_{O} \leq \gamma|w|_{I}, \quad \forall w \in I
$$

Let $u \in C_{0 c}\left(\mathbb{R}_{+}, X\right)$ and let

$$
\psi_{u}: \mathbb{R}_{+} \longrightarrow \mathbb{R}, \quad \psi_{u}(t)=\int_{0}^{t} e^{-v(t-s)}\|u(s)\| d s .
$$

Then for every $\theta \in \Theta$ we have that

$$
\left\|x_{\theta}(t ; 0, u)\right\| \leq K \psi_{u}(t), \quad t \geq 0 .
$$

Case 1. $I \in \mathcal{R}\left(\mathbb{R}_{+}\right)$Let $\lambda_{I}>0$ be given by Lemma 2.17. Since $u \in I\left(\mathbb{R}_{+}, X\right)$, from Lemma 2.17 we have that $\psi_{u} \in I$ and $\left|\psi_{u}\right|_{I} \leq \lambda_{I}\|u\|_{I\left(\mathbb{R}_{+}, X\right)}$. Then from (3.27) it follows that $x_{\theta}(\cdot ; 0, u) \in I\left(\mathbb{R}_{+}, X\right)$, so $x_{\theta}(\cdot ; 0, u) \in O\left(\mathbb{R}_{+}, X\right)$. Moreover, from (3.25) and (3.27) we have that

$$
\left\|x_{\theta}(\cdot ; 0, u)\right\|_{O\left(\mathbb{R}_{+}, X\right)} \leq \gamma\left\|x_{\theta}(\cdot ; 0, u)\right\|_{I\left(\mathbb{R}_{+}, X\right)} \leq \gamma K \lambda_{I}\|u\|_{I\left(\mathbb{R}_{+}, X\right)} .
$$

Taking $\lambda=\gamma K \lambda_{I}$ and observing that $\lambda$ does not depend on $u$ or $\theta$ we deduce that the system $(S)$ is $\left(I\left(\mathbb{R}_{+}, X\right), O\left(\mathbb{R}_{+}, X\right)\right)$ stable. 
Case 2. $O \in \mathcal{R}\left(\mathbb{R}_{+}\right)$Let $\lambda_{O}>0$ be given by Lemma 2.17. From $u \in O\left(\mathbb{R}_{+}, X\right)$, using Lemma 2.17 we have that $\psi_{u} \in O$ and $\left|\psi_{u}\right|_{O} \leq \lambda_{O}\|u\|_{O\left(\mathbb{R}_{+}, X\right)}$. Then, from (3.25) and (3.27) we deduce that $x_{\theta}(\cdot ; 0, u) \in O\left(\mathbb{R}_{+}, X\right)$ and

$$
\left\|x_{\theta}(\cdot ; 0, u)\right\|_{O\left(\mathbb{R}_{+}, X\right)} \leq K \lambda_{O}\|u\|_{O\left(\mathbb{R}_{+}, X\right)} \leq \gamma K \lambda_{O}\|u\|_{I\left(\mathbb{R}_{+}, X\right)} .
$$

Taking $\lambda=\gamma K \lambda_{O}$ we conclude that the system $(S)$ is $\left(I\left(\mathbb{R}_{+}, X\right), O\left(\mathbb{R}_{+}, X\right)\right)$ stable.

Sufficiency. It follows from (i).

In what follows, we prove that the main result given by Theorem 3.9 is the most general in this topic. Specifically, we will show that if $I \notin \mathcal{L}\left(\mathbb{R}_{+}\right)$and $O \notin \mathcal{Q}\left(\mathbb{R}_{+}\right)$, then the $\left(I\left(\mathbb{R}_{+}, X\right), O\left(\mathbb{R}_{+}, X\right)\right)$ stability of the system $(S)$ does not imply the uniform exponential stability of $(S)$.

Example 3.10. Let $\Theta=\mathbb{R}_{+}$and let $\sigma: \Theta \times \mathbb{R}_{+} \rightarrow \Theta, \sigma(\theta, t)=\theta+t$. Let $X=C_{0}\left(\mathbb{R}_{+}, \mathbb{R}\right)$ and for every $(\theta, t) \in \Theta \times \mathbb{R}_{+}$, let

$$
\Phi(\theta, t): X \longrightarrow X, \quad(\Phi(\theta, t) x)(s)=x(s+t)
$$

Then $\pi=(\Phi, \sigma)$ is a linear skew-product flow on $\varepsilon=X \times \Theta$. We consider the system

$$
x_{\theta}\left(t ; x_{0}, u\right)=\Phi(\theta, t) x_{0}+\int_{0}^{t} \Phi(\sigma(\theta, s), t-s) u(s) d s, \quad t \geq 0, \theta \in \Theta
$$

with $u \in L_{\text {loc }}^{1}\left(\mathbb{R}_{+}, X\right)$ and $x_{0} \in X$. Observing that $\|\Phi(\theta, t)\|=1$, for all $(\theta, t) \in \Theta \times \mathbb{R}_{+}$, we deduce that the system $(S)$ is not uniformly exponentially stable.

Let $I, O \in \tau\left(\mathbb{R}_{+}\right)$with $I \notin \mathcal{L}\left(\mathbb{R}_{+}\right)$and $O \notin Q\left(\mathbb{R}_{+}\right)$. Then $I \subset L^{1}\left(\mathbb{R}_{+}, \mathbb{R}\right)$ and from Lemma 2.19 we have that $C_{0}\left(\mathbb{R}_{+}, \mathbb{R}\right) \subset O$. In what follows, we prove that the system $(S)$ is $\left(I\left(\mathbb{R}_{+}, X\right), O\left(\mathbb{R}_{+}, X\right)\right)$ stable.

Let $u \in L^{1}\left(\mathbb{R}_{+}, X\right)$ and $\theta \in \Theta$. Let $\varepsilon>0$. Since $u \in L^{1}\left(\mathbb{R}_{+}, X\right)$, there is $h>0$ such that $\int_{h}^{\infty}\|u(s)\| d s<\varepsilon / 2$. Let $v:=\int_{0}^{h} \Phi(\sigma(\theta, \tau), h-\tau) u(\tau) d \tau$. From $v \in X$ we have that there is $\delta>0$ such that $|v(\tau)|<\varepsilon / 2$, for all $t \geq \delta$. Then, for $t \geq \delta+h$, we deduce that

$$
\left\|\int_{0}^{h} \Phi(\sigma(\theta, s), t-s) u(s) d s\right\|=\|\Phi(\sigma(\theta, h), t-h) v\|=\sup _{r \geq 0}|v(t-h+r)|<\frac{\varepsilon}{2} .
$$

This implies that

$$
\begin{aligned}
\left\|x_{\theta}(t ; 0, u)\right\| & \leq\left\|\int_{0}^{h} \Phi(\sigma(\theta, s), t-s) u(s) d s\right\|+\int_{h}^{t}\|\Phi(\sigma(\theta, s), t-s)\|\|u(s)\| d s \\
& <\frac{\varepsilon}{2}+\int_{h}^{t}\|u(s)\| d s<\varepsilon, \quad \forall t \geq \delta+h
\end{aligned}
$$

so $x_{\theta}(\cdot ; 0, u) \in C_{0}\left(\mathbb{R}_{+}, X\right)$. It follows that for every $(u, \theta) \in L^{1}\left(\mathbb{R}_{+}, X\right) \times \Theta$ we have that $x_{\theta}(\cdot ; 0, u) \in$ $C_{0}\left(\mathbb{R}_{+}, X\right)$. 
It makes sense to define the linear operator

$$
P: L^{1}\left(\mathbb{R}_{+}, X\right) \longrightarrow C_{0}\left(\mathbb{R}_{+}, X\right), \quad P(u)=x_{0}(\cdot ; 0, u)
$$

It is easy to verify that $P$ is closed, so from the closed graph theorem we obtain that $P$ is bounded. Setting $\gamma=\|P\|$ we have that

$$
\left\|x_{0}(\cdot ; 0, u)\right\|_{C_{0}\left(\mathbb{R}_{+}, X\right)} \leq r\|u\|_{L^{1}\left(\mathbb{R}_{+}, X\right)}, \quad \forall u \in L^{1}\left(\mathbb{R}_{+}, X\right) .
$$

Since $I \subset L^{1}\left(\mathbb{R}_{+}, \mathbb{R}\right)$ there is $\alpha>0$ such that

$$
\|u\|_{1} \leq \alpha|u|_{I}, \quad \forall u \in I .
$$

Since $C_{0}\left(\mathbb{R}_{+}, \mathbb{R}\right) \subset O$ there is $\beta>0$ such that

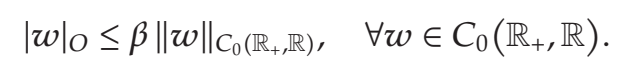

Let $(u, \theta) \in C_{0 c}\left(\mathbb{R}_{+}, X\right) \times \Theta$. Since $x_{\theta}(\cdot ; 0, u) \in C_{0}\left(\mathbb{R}_{+}, X\right)$ and $C_{0}\left(\mathbb{R}_{+}, X\right) \subset O\left(\mathbb{R}_{+}, X\right)$ it follows that $x_{\theta}(\cdot ; 0, u) \in O\left(\mathbb{R}_{+}, X\right)$. It is easy to observe that $x_{\theta}(\cdot ; 0, u)=x_{0}(\cdot ; 0, u)$. Setting $\lambda=$ $\alpha \beta \gamma$, from relations (3.34)-(3.36) we deduce that

$$
\left\|x_{\theta}(\cdot ; 0, u)\right\|_{O\left(\mathbb{R}_{+}, X\right)}=\left\|x_{0}(\cdot ; 0, u)\right\|_{O\left(\mathbb{R}_{+}, X\right)} \leq \beta\left\|x_{0}(\cdot ; 0, u)\right\|_{C_{0}\left(\mathbb{R}_{+}, X\right)} \leq \beta \gamma\|u\|_{L^{1}\left(\mathbb{R}_{+}, X\right)} \leq \lambda\|u\|_{I\left(\mathbb{R}_{+}, X\right)}
$$

Taking into account that $\lambda$ does not depend on $u$ or $\theta$ we conclude that the system $(S)$ is $\left(I\left(\mathbb{R}_{+}, X\right), O\left(\mathbb{R}_{+}, X\right)\right)$ stable. But, for all that $(S)$ is not uniformly exponentially stable.

Remark 3.11. The input-output characterizations for asymptotic properties of systems have a wide applicability area if the input space is as small as possible and the output space is very general. We note that in the main result given by Theorem 3.9 the input functions belong to $C_{0 c}\left(\mathbb{R}_{+}, X\right)$, while the output space is a general function space.

Moreover, the class $\tau\left(\mathbb{R}_{+}\right)$is closed to finite intersections. If $I_{1}, I_{2}, \ldots, I_{n} \in \tau\left(\mathbb{R}_{+}\right)$, then we may consider the space $I:=I_{1} \cap I_{2} \cap \cdots \cap I_{n}$ with respect to the norm

$$
|u|_{I}:=\max \left\{|u|_{I_{1}},|u|_{I_{2}}, \ldots,|u|_{I_{n}}\right\}
$$

which is a Banach function space in $\tau\left(\mathbb{R}_{+}\right)$. Now, if we analyze condition (ii) in Definition 3.5, it is obvious that if the input space is $I\left(\mathbb{R}_{+}, X\right)$, then in the right member of the inequality we have a "larger" norm and thus the estimation is more permissive. 
Let $q \in[1, \infty], n \in \mathbb{N}^{*}$, and $p_{1}, \ldots, p_{n} \in(1, \infty)$. We consider the space

$$
I^{p_{1}, \ldots, p_{n}, q}\left(\mathbb{R}_{+}, \mathbb{R}\right)=L^{p_{1}}\left(\mathbb{R}_{+}, \mathbb{R}\right) \cap \cdots \cap L^{p_{n}}\left(\mathbb{R}_{+}, \mathbb{R}\right) \cap L^{q}\left(\mathbb{R}_{+}, \mathbb{R}\right)
$$

which is a Banach space with respect the norm

$$
\|u\|_{p_{1}, \ldots, p_{n}, q}=\max \left\{\|u\|_{p_{1}}, \ldots,\|u\|_{p_{n}},\|u\|_{q}\right\} .
$$

Corollary 3.12. The system $(S)$ is uniformly exponentially stable if and only if the system $(S)$ is $\left(I^{p_{1}, \ldots, p_{n}, q}\left(\mathbb{R}_{+}, X\right), L^{q}\left(\mathbb{R}_{+}, X\right)\right)$ stable.

Proof. If $q \in[1, \infty)$, then $L^{q}\left(\mathbb{R}_{+}, \mathbb{R}\right) \in \mathcal{Q}\left(\mathbb{R}_{+}\right)$. If $q=\infty$, then $I \in \mathcal{L}\left(\mathbb{R}_{+}\right)$. By applying Theorem 3.9 we obtain the conclusion.

Corollary 3.13. Let $W \in R\left(\mathbb{R}_{+}\right)$. The system (S) is uniformly exponentially stable if and only if $(S)$ is $\left(W\left(\mathbb{R}_{+}, X\right), W\left(\mathbb{R}_{+}, X\right)\right)$ stable.

Proof. This follows from Theorem 3.9 and Lemma 2.21.

Lemma 3.14. Let $v>0$ and $p, q \in[1, \infty]$ with $p \leq q$. For every $u \in L^{p}\left(\mathbb{R}_{+}, \mathbb{R}\right)$, the function

$$
f_{u}: \mathbb{R}_{+} \longrightarrow \mathbb{R}, \quad f_{u}(t)=\int_{0}^{t} e^{-v(t-s)} u(s) d s
$$

belongs to $L^{q}\left(\mathbb{R}_{+}, \mathbb{R}\right)$. In addition, there is $\gamma>0$ such that $\left\|f_{u}\right\|_{q} \leq \gamma\|u\|_{p}$, for all $u \in L^{p}\left(\mathbb{R}_{+}, X\right)$.

Proof. Using Hölder's inequality it follows that for every $u \in L^{p}\left(\mathbb{R}_{+}, \mathbb{R}_{+}\right)$the function $f_{u} \in$ $L^{q}\left(\mathbb{R}_{+}, \mathbb{R}_{+}\right)$. Then, from

$$
\left|f_{u}(t)\right| \leq \int_{0}^{t} e^{-v(t-s)}|u(s)| d s, \quad \forall t \geq 0, \forall u \in L^{p}\left(\mathbb{R}_{+}, \mathbb{R}\right)
$$

we deduce that for every $u \in L^{p}\left(\mathbb{R}_{+}, \mathbb{R}\right)$ the function $f_{u}$ belongs to $L^{q}\left(\mathbb{R}_{+}, \mathbb{R}\right)$. Hence, it makes sense to define the linear operator $\Gamma: L^{p}\left(\mathbb{R}_{+}, \mathbb{R}\right) \rightarrow L^{q}\left(\mathbb{R}_{+}, \mathbb{R}\right), \Gamma(u)=f_{u}$. It is easy to see that $\Gamma$ is closed, so it is bounded. Setting $\gamma=\|\Gamma\|$ we obtain the conclusion.

Corollary 3.15. Let $p, q \in[1, \infty]$ with $(p, q) \neq(1, \infty)$. The following assertions hold:

(i) if the system $(S)$ is $\left(L^{p}\left(\mathbb{R}_{+}, X\right), L^{q}\left(\mathbb{R}_{+}, X\right)\right)$ stable, then $(S)$ is uniformly exponentially stable;

(ii) if $p \leq q$, then the system (S) is uniformly exponentially stable if and only if it is $\left(L^{p}\left(\mathbb{R}_{+}, X\right), L^{q}\left(\mathbb{R}_{+}, X\right)\right)$ stable.

Proof. (i) It follows from Theorem 3.9(i).

(ii) Necessity. Let $\gamma>0$ be given by Lemma 3.14. Let $K, v>0$ be such that $\|\Phi(\theta, t) x\| \leq$ $K e^{-v t}\|x\|$, for all $(x, \theta) \in \mathcal{E}$ and all $t \geq 0$. Let $(u, \theta) \in C_{0 c}\left(\mathbb{R}_{+}, X\right) \times \Theta$. Then

$$
\left\|x_{\theta}(t ; 0, u)\right\| \leq K \int_{0}^{t} e^{-v(t-s)}\|u(s)\| d s, \quad \forall t \geq 0 .
$$


Using Lemma 3.14 we obtain that $x_{\theta}(\cdot ; 0, u) \in L^{q}\left(\mathbb{R}_{+}, X\right)$ and

$$
\left\|x_{\theta}(\cdot ; 0, u)\right\|_{L^{q}\left(\mathbb{R}_{+}, X\right)} \leq K \gamma\|u\|_{L^{p}\left(\mathbb{R}_{+}, X\right)} .
$$

Taking into account that $\gamma$ and $K$ do not depend on $u$ or $\theta$, it follows that the system (S) is $\left(L^{p}\left(\mathbb{R}_{+}, X\right), L^{q}\left(\mathbb{R}_{+}, X\right)\right)$ stable.

Sufficiency. This follows from (i).

Definition 3.16. Let $I, O$ be two Banach function spaces with $I, O \in \tau\left(\mathbb{R}_{+}\right)$. The system $(S)$ is said to be completely $\left(I\left(\mathbb{R}_{+}, X\right), O\left(\mathbb{R}_{+}, X\right)\right)$ stable if the following assertions hold:

(i) for every $u \in I\left(\mathbb{R}_{+}, X\right)$ and every $\theta \in \Theta$ the solution $x_{\theta}(; 0, u) \in O\left(\mathbb{R}_{+}, X\right)$;

(ii) there is $\lambda>0$ such that $\left\|x_{\theta}(\cdot ; 0, u)\right\|_{O\left(\mathbb{R}_{+}, X\right)} \leq \lambda\|u\|_{I\left(\mathbb{R}_{+}, X\right)}$, for all $(u, \theta) \in I\left(\mathbb{R}_{+}, X\right) \times \Theta$.

Theorem 3.17. Let $I, O \in \tau\left(\mathbb{R}_{+}\right)$be such that $I \in \mathcal{L}\left(\mathbb{R}_{+}\right)$or $O \in \mathcal{Q}\left(\mathbb{R}_{+}\right)$. Then, the following assertions hold:

(i) if the system $(S)$ is completely $\left(I\left(\mathbb{R}_{+}, X\right), O\left(\mathbb{R}_{+}, X\right)\right)$ stable, then $(S)$ is uniformly exponentially stable;

(ii) if $I \subset O$ and one of the spaces $I, O$ belongs to the class $R\left(\mathbb{R}_{+}\right)$, then the system (S) is uniformly exponentially stable if and only if the system $(S)$ is completely $\left(I\left(\mathbb{R}_{+}, X\right), O\left(\mathbb{R}_{+}, X\right)\right)$ stable.

Proof. (i) This follows from Theorem 3.9(i).

(ii) Necessity follows using similar arguments as in the necessity part of Theorem 3.9(ii). Sufficiency is given by (i).

Corollary 3.18. Let $q \in[1, \infty], n \in \mathbb{N}^{*}$ and $p_{1}, \ldots, p_{n} \in(1, \infty)$. The system $(S)$ is uniformly exponentially stable if and only if the system $(S)$ is completely $\left(I^{p_{1}, \ldots, p_{n}, q}\left(\mathbb{R}_{+}, X\right), L^{q}\left(\mathbb{R}_{+}, X\right)\right)$ stable.

Proof. This follows from Corollary 3.12.

Corollary 3.19. Let $W \in R\left(\mathbb{R}_{+}\right)$. The system (S) is uniformly exponentially stable if and only if $(S)$ is completely $\left(W\left(\mathbb{R}_{+}, X\right), W\left(\mathbb{R}_{+}, X\right)\right)$ stable.

Remark 3.20. Let $W \in \mathcal{R}\left(\mathbb{R}_{+}\right)$. If the system $(S)$ is uniformly exponentially stable, then for every $\theta \in \Theta$ the linear operator

$$
P_{W}^{\theta}: W\left(\mathbb{R}_{+}, X\right) \longrightarrow W\left(\mathbb{R}_{+}, X\right), \quad\left(P_{W}^{\theta} u\right)(t)=\int_{0}^{t} \Phi(\sigma(\theta, s), t-s) u(s) d s
$$

is correctly defined and bounded. Moreover, if $\lambda>0$ is given by Definition 3.16, then we have that $\sup _{\theta \in \Theta}\left\|P_{W}^{\theta}\right\| \leq \lambda$.

Corollary 3.21. Let $p, q \in[1, \infty]$ with $(p, q) \neq(1, \infty)$. The following assertions hold:

(i) if the system $(S)$ is completely $\left(L^{p}\left(\mathbb{R}_{+}, X\right), L^{q}\left(\mathbb{R}_{+}, X\right)\right)$ stable, then $(S)$ is uniformly exponentially stable;

(ii) if $p \leq q$, then the system (S) is uniformly exponentially stable if and only if it is completely $\left(L^{p}\left(\mathbb{R}_{+}, X\right), L^{q}\left(\mathbb{R}_{+}, X\right)\right)$ stable. 
Proof. (i) This follows from Corollary 3.15(i).

(ii) Necessity follows using similar arguments as in the necessity of Corollary 3.15(ii). Sufficiency follows from (i).

Remark 3.22. A distinct proof for Corollary 3.21(i) was given in [27] (see Theorem 5.2).

Let $C_{b}\left(\mathbb{R}_{+}, \mathbb{R}\right)$ be the space of all bounded continuous functions $u: \mathbb{R}_{+} \rightarrow \mathbb{R}$ and $C_{00}\left(\mathbb{R}_{+}, \mathbb{R}\right)=\left\{u \in C_{0}\left(\mathbb{R}_{+}, \mathbb{R}\right): u(0)=0\right\}$.

Definition 3.23. Let $U, Y \in\left\{C_{b}\left(\mathbb{R}_{+}, \mathbb{R}\right), C_{0}\left(\mathbb{R}_{+}, \mathbb{R}\right), C_{00}\left(\mathbb{R}_{+}, \mathbb{R}\right)\right\}$. The system $(S)$ is said to be completely $\left(U\left(\mathbb{R}_{+}, X\right), Y\left(\mathbb{R}_{+}, X\right)\right)$ stable if the following assertions hold:

(i) for every $u \in U\left(\mathbb{R}_{+}, X\right)$ and every $\theta \in \Theta$ the solution $x_{\theta}(\cdot ; 0, u) \in Y\left(\mathbb{R}_{+}, X\right)$;

(i) there is $\lambda>0$ such that $\left\|x_{\theta}(\cdot ; 0, u)\right\|_{Y\left(\mathbb{R}_{+}, X\right)} \leq \lambda\|u\|_{U\left(\mathbb{R}_{+}, X\right)}$, for all $(u, \theta) \in U\left(\mathbb{R}_{+}, X\right) \times \Theta$.

Corollary 3.24. Let $U, Y \in\left\{C_{b}\left(\mathbb{R}_{+}, \mathbb{R}\right), C_{0}\left(\mathbb{R}_{+}, \mathbb{R}\right), C_{00}\left(\mathbb{R}_{+}, \mathbb{R}\right)\right\}$ with $U \subset Y$. The system (S) is uniformly exponentially stable if and only if $(S)$ is completely $\left(U\left(\mathbb{R}_{+}, X\right), Y\left(\mathbb{R}_{+}, X\right)\right)$ stable.

Proof. Necessity is a simple exercise.

Sufficiency. If the system $(S)$ is completely $\left(U\left(\mathbb{R}_{+}, X\right), Y\left(\mathbb{R}_{+}, X\right)\right)$ stable, then it is $\left(L^{\infty}\left(\mathbb{R}_{+}, X\right), L^{\infty}\left(\mathbb{R}_{+}, X\right)\right)$ stable. By applying Corollary 3.15 we deduce that $(S)$ is uniformly exponentially stable.

Remark 3.25. A different proof for Corollary 3.24 for the cases $(U, Y)=\left(C_{00}\left(\mathbb{R}_{+}, \mathbb{R}\right), C_{00}\left(\mathbb{R}_{+}, \mathbb{R}\right)\right)$ and $(U, Y)=\left(C_{00}\left(\mathbb{R}_{+}, \mathbb{R}\right), C_{b}\left(\mathbb{R}_{+}, \mathbb{R}\right)\right)$ was given in [27] (see Theorem 5.1).

\section{Stability radius}

In this section, we will obtain a lower bound for the stability radius of linear skew-product flows in terms of input-output operators acting on Banach function spaces which belong to a class of rearrangement invariant spaces.

Let $U, Y$ be Banach spaces and let $\Theta$ be a locally compact metric space. We denote by $B(U, Y)$ the space of all bounded linear operators from $U$ into $Y$ and by $\mathcal{C}_{s}(\Theta, B(U, Y))$ the space of all continuous bounded mappings $H: \Theta \rightarrow B(U, Y)$. With respect to the norm $\|H\| \mid=$ $\sup _{\theta \in \Theta}\|H(\theta)\|, \mathcal{C}_{S}(\Theta, B(U, Y))$ is a Banach space.

Remark 4.1. If $\pi=(\Phi, \sigma)$ is a linear skew-product flow on $\varepsilon=X \times \Theta$ and $P \in \mathcal{C}_{s}(\Theta, B(X))$, then there exists a unique linear skew-product flow denoted $\pi_{P}=\left(\Phi_{P}, \sigma\right)$ on $X \times \Theta$ such that

$$
\Phi_{P}(\theta, t) x=\Phi(\theta, t) x+\int_{0}^{t} \Phi(\sigma(\theta, s), t-s) P(\sigma(\theta, s)) \Phi_{P}(\theta, s) x d s
$$

for all $(x, \theta, t) \in X \times \Theta \times \mathbb{R}_{+}$(see [26, Theorem 2.1]).

Let $X$ be a Banach space, let $\Theta$ be a locally compact metric space, and let $\pi=(\Phi, \sigma)$ be a linear skew-product flow on $\mathcal{\varepsilon}=X \times \Theta$. We consider the variational integral control system

$$
x_{\theta}\left(t ; x_{0}, u\right)=\Phi(\theta, t) x_{0}+\int_{0}^{t} \Phi(\sigma(\theta, s), t-s) u(s) d s, \quad t \geq 0, \theta \in \Theta
$$

with $u \in L_{\mathrm{loc}}^{1}\left(\mathbb{R}_{+}, X\right)$ and $x_{0} \in X$. 
For every $P \in \mathcal{C}_{S}(\Theta, \mathcal{B}(X))$ we consider the perturbed system

$$
x_{\theta}\left(t ; x_{0}, u\right)=\Phi_{P}(\theta, t) x_{0}+\int_{0}^{t} \Phi_{P}(\sigma(\theta, s), t-s) u(s) d s, \quad t \geq 0, \theta \in \Theta
$$

with $u \in L_{\mathrm{loc}}^{1}\left(\mathbb{R}_{+}, X\right)$ and $x_{0} \in X$.

Let $U, Y$ be Banach spaces, let $B \in \mathcal{C}_{s}(\Theta, B(U, X))$ and let $C \in \mathcal{C}_{s}(\Theta, B(X, Y))$. We consider the system $(S, B, C)$ described by the following integral model:

$$
\begin{gathered}
x_{\theta}\left(t, x_{0}, u\right)=\Phi(\theta, t) x_{0}+\int_{0}^{t} \Phi(\sigma(\theta, s), t-s) B(\sigma(\theta, s)) u(s) d s, \quad t \geq 0, \theta \in \Theta, \\
y_{\theta}\left(t, x_{0}, u\right)=C(\sigma(\theta, t)) x_{\theta}\left(t, x_{0}, u\right), \quad t \geq 0, \theta \in \Theta,
\end{gathered}
$$

where $x_{0} \in X$ and $u \in L_{\text {loc }}^{1}\left(\mathbb{R}_{+}, U\right)$.

Throughout this section, we suppose that $(S)$ is uniformly exponentially stable. The stability radius of $(S)$ with respect to the perturbation structure $(B, C)$ is defined by

$$
\begin{aligned}
r_{\text {stab }}(S, B, C)=\sup \{r & \geq 0: \forall \Delta \in \mathcal{C}_{S}(\Theta, B(Y, U)) \text { with }\||\Delta \|| \leq r \\
& \Longrightarrow \text { the system }(S, B \Delta C) \text { is uniformly exponentially stable }\}
\end{aligned}
$$

Let $\mho\left(\mathbb{R}_{+}\right)$be the class of all Banach function spaces $B \in \mathcal{R}\left(\mathbb{R}_{+}\right)$with the property that for every $u \in B,\left|u \chi_{[0, n)}\right|_{B} \rightarrow|u|_{B}$ as $n \rightarrow \infty$.

Remark 4.2. It is easy to verify that the Orlicz spaces belong to the class $\mho\left(\mathbb{R}_{+}\right)$.

Let $V \in \mathcal{U}\left(\mathbb{R}_{+}\right)$. Since the system $(S)$ is uniformly exponentially stable from Remark 3.20 we have that for every $\theta \in \Theta$, the linear operators

$$
P_{V}^{\theta}: V\left(\mathbb{R}_{+}, X\right) \longrightarrow V\left(\mathbb{R}_{+}, X\right), \quad\left(P_{V}^{\theta} u\right)(t)=\int_{0}^{t} \Phi(\sigma(\theta, s), t-s) u(s) d s
$$

are bounded and $\sup _{\theta \in \Theta}\left\|P_{V}^{\theta}\right\|<\infty$.

Lemma 4.3. For every $\theta \in \Theta$, the linear operators,

$$
\begin{array}{ll}
B_{V}^{\theta}: V\left(\mathbb{R}_{+}, U\right) \longrightarrow V\left(\mathbb{R}_{+}, X\right), & \left(B_{V}^{\theta} u\right)(t)=B(\sigma(\theta, t)) u(t), \\
C_{V}^{\theta}: V\left(\mathbb{R}_{+}, X\right) \longrightarrow V\left(\mathbb{R}_{+}, Y\right), & \left(C_{V}^{\theta} u\right)(t)=C(\sigma(\theta, t)) u(t),
\end{array}
$$

are bounded. Moreover, $\sup _{\theta \in \Theta}\left\|B_{V}^{\theta}\right\| \leq\|B\| \mid$ and $\sup _{\theta \in \Theta}\left\|C_{V}^{\theta}\right\| \leq\|\mid C\|$.

Proof. Let $\theta \in \Theta$. For every $u \in V\left(\mathbb{R}_{+}, U\right)$ we have that

$$
\left\|\left(B_{V}^{\theta} u\right)(t)\right\| \leq\|B(\sigma(\theta, t))\|\|u(t)\| \leq\|\mid B\|\|\| u(t) \|, \quad \forall t \geq 0
$$

which implies that $\left\|B_{V}^{\theta} u\right\|_{V\left(\mathbb{R}_{+}, X\right)} \leq\|\mid B\|\|\| u \|_{V\left(\mathbb{R}_{+}, U\right)}$. This shows that $\left\|B_{V}^{\theta}\right\| \leq\|B\|$, for all $\theta \in \Theta$. Similarly, we obtain that $\left\|C_{V}^{\theta}\right\| \leq\||C \||$, for all $\theta \in \Theta$. 
Definition 4.4. For every $\theta \in \Theta$, we consider the bounded linear operators

$$
L_{V}^{\theta}: V\left(\mathbb{R}_{+}, U\right) \longrightarrow V\left(\mathbb{R}_{+}, Y\right), \quad L_{V}^{\theta}=C_{V}^{\theta} P_{V}^{\theta} B_{V}^{\theta} .
$$

The family $\left\{L_{V}^{\theta}\right\}_{\theta \in \Theta}$ is called the family of input-output operators associated to the system $(S, B, C)$. In all what follows we denote

$$
\alpha_{V}(S, B, C):=\sup _{\theta \in \Theta}\left\|L_{V}^{\theta}\right\|
$$

Remark 4.5. Using Remark 3.20 and Lemma 4.3 we have that $\alpha_{V}(S, B, C)<\infty$.

For every $\Delta \in \mathcal{C}_{s}(\Theta, B(Y, U))$ and every $\theta \in \Theta$, we consider the bounded linear operator

$$
\Delta_{V}^{\theta}: V\left(\mathbb{R}_{+}, Y\right) \longrightarrow V\left(\mathbb{R}_{+}, U\right), \quad\left(\Delta_{V}^{\theta} u\right)(t)=\Delta(\sigma(\theta, t)) u(t) .
$$

Then we have that $\left\|\Delta_{V}^{\theta}\right\| \leq\||\Delta \||$, for all $\theta \in \Theta$.

In what follows we suppose that there is $m>0$ such that $\|C(\theta) x\|_{Y} \geq m\|x\|_{X}$, for all $(x, \theta) \in X \times \Theta$.

Theorem 4.6. Let $\Delta \in \mathcal{C}_{s}(\Theta, B(Y, U))$ with $\left\||\Delta \||<1 / \alpha_{V}(S, B, C)\right.$. Then, for every $(x, \theta) \in X \times \Theta$, the function

$$
f_{x, \theta}: \mathbb{R}_{+} \longrightarrow X, \quad f_{x, \theta}(t)=\Phi_{B \Delta C}(\theta, t) x
$$

belongs to $V\left(\mathbb{R}_{+}, X\right)$.

Proof. Let $\gamma_{V}=\alpha_{V}(S, B, C)\||\Delta \||$. Let $K, v>0$ be such that $\| \Phi(\theta, t) \| \leq K e^{-v t}$, for all $(\theta, t) \in$ $\Theta \times \mathbb{R}_{+}$.

Let $(x, \theta) \in \mathcal{E}$. For every $n \in \mathbb{N}^{*}$ let

$$
\begin{aligned}
f_{n}: \mathbb{R}_{+} \longrightarrow X, & f_{n}(t)=X[0, n)(t) f_{x, \theta}(t), \\
g_{n}: \mathbb{R}_{+} \longrightarrow X, & g_{n}(t)=C(\sigma(\theta, t)) f_{n}(t) .
\end{aligned}
$$

Using Remark 4.1, we have that $f_{n}(t)=\chi_{[0, n)}(t) \Phi(\theta, t) x+\left(P_{V}^{\theta} B_{V}^{\theta} \Delta_{V}^{\theta} g_{n}\right)(t)$, for all $t \in[0, n)$, which implies that

$$
g_{n}(t)=\chi_{[0, n)}(t) C(\sigma(\theta, t)) \Phi(\theta, t) x+\left(L_{V}^{\theta} \Delta_{V}^{\theta} g_{n}\right)(t), \quad \forall t \in[0, n) .
$$

Then, for $t \in[0, n)$ we have that

$$
\left\|g_{n}(t)\right\|_{Y} \leq\left\|\left|C \left\|\left|K\|x\| e^{-v t}+\left\|L_{V}^{\theta}\right\|\left\|\Delta_{V}^{\theta}\right\|\left\|g_{n}(t)\right\|_{Y} \leq\left\|\left|C\|\mid K\| x\left\|e^{-v t}+\gamma_{V}\right\| g_{n}(t) \|_{Y}\right.\right.\right.\right.\right.\right.
$$

Since $g_{n}(t)=0$ for $t \geq n$, we deduce that

$$
\left\|g_{n}(t)\right\|_{Y} \leq\|C\| \mid K\|x\| e^{-v t}+\gamma_{V}\left\|g_{n}(t)\right\|_{\gamma^{\prime}} \quad \forall t \geq 0 .
$$


Let $e_{v}: \mathbb{R}_{+} \rightarrow \mathbb{R}_{+}, e_{v}(t)=e^{-v t}$. From $V \in \mathcal{U}\left(\mathbb{R}_{+}\right)$and using Lemma 2.12 we have that $e_{v} \in V$. Then, from (4.14) it follows that

$$
\left\|g_{n}\right\|_{V\left(\mathbb{R}_{+}, Y\right)} \leq \delta_{x}\left|e_{\nu}\right|_{V}+\gamma_{V}\left\|g_{n}\right\|_{V\left(\mathbb{R}_{+}, Y\right)^{\prime}}
$$

where $\delta_{x}=\||C\|\mid K\| x \|$. The above inequality shows that

$$
\left\|g_{n}\right\|_{V\left(\mathbb{R}_{+}, Y\right)} \leq \frac{\delta_{x}\left|e_{\nu}\right|_{V}}{1-\gamma_{V}}, \quad \forall n \in \mathbb{N}^{*}
$$

Since $V \in \mathcal{U}\left(\mathbb{R}_{+}\right)$from (4.16) we deduce that the function $g_{x, \theta}: \mathbb{R}_{+} \rightarrow Y, g_{x, \theta}(t)=$ $C(\sigma(\theta, t)) f_{x, \theta}(t)$ belongs to $V\left(\mathbb{R}_{+}, Y\right)$. Then, from $m\left\|f_{x, \theta}(t)\right\|_{X} \leq\left\|g_{x, \theta}(t)\right\|_{Y}$, for all $t \geq 0$, we obtain that $f_{x, \theta} \in V\left(\mathbb{R}_{+}, X\right)$.

Theorem 4.7. The following estimation holds:

$$
r_{\mathrm{stab}}(S, B, C) \geq \frac{1}{\alpha_{V}(S, B, C)}
$$

Proof. Let $\Delta \in \mathcal{C}_{S}(\Theta, \mathbb{B}(Y, U))$ be such that $\left\||\Delta \||<1 / \alpha_{V}(S, B, C)\right.$. Let $\left.\gamma_{V}=\alpha_{V}(S, B, C)\right\||\Delta \||$.

We prove that the perturbed system $(S, B \Delta C)$ is completely $\left(V\left(\mathbb{R}_{+}, X\right), V\left(\mathbb{R}_{+}, X\right)\right)$ stable. Let $v \in V\left(\mathbb{R}_{+}, X\right)$ and $\theta \in \Theta$. The corresponding solution of the system $(S, B \Delta C)$ is

$$
x_{\theta}(\cdot ; 0, v): \mathbb{R}_{+} \longrightarrow X, \quad x_{\theta}(t ; 0, v)=\int_{0}^{t} \Phi_{B \Delta C}(\sigma(\theta, s), t-s) v(s) d s
$$

Let $n \in \mathbb{N}^{*}$. We set $v_{n}=v \chi_{[0, n)}$ and $x_{n}=x_{\theta}(n ; 0, v)$. Then, we have that

$$
x_{\theta}\left(t ; 0, v_{n}\right)=\Phi_{B \Delta C}(\sigma(\theta, n), t-n) x_{n}, \quad \forall t \geq n .
$$

From Theorem 4.6 we have that the function

$$
\varphi: \mathbb{R}_{+} \longrightarrow X, \quad \varphi(t)=\Phi(\sigma(\theta, n), t) x_{n}
$$

belongs to $V\left(\mathbb{R}_{+}, X\right)$. Since $V$ is invariant to translations, it follows that

$$
\psi: \mathbb{R}_{+} \longrightarrow X, \quad \psi(t)= \begin{cases}\Phi(\sigma(\theta, n), t-n) x_{n}, & t \geq n, \\ 0, & t \in[0, n),\end{cases}
$$

belongs to $V\left(\mathbb{R}_{+}, X\right)$.

Since $x_{\theta}\left(\cdot ; 0, v_{n}\right)$ is continuous, setting $M_{n}=\sup _{t \in[0, n]}\left\|x_{\theta}\left(t ; 0, v_{n}\right)\right\|$ and using (4.19) we deduce that

$$
\left\|x_{\theta}\left(t ; 0, v_{n}\right)\right\| \leq M_{n} \chi_{[0, n)}(t)+\|\psi(t)\|, \quad \forall t \geq 0
$$

which implies that $x_{\theta}\left(\cdot ; 0, v_{n}\right) \in V\left(\mathbb{R}_{+}, X\right)$. 
Using Remark 4.1 and Fubini's theorem we deduce that

$$
\left(C_{V}^{\theta} x_{\theta}\left(\cdot ; 0, v_{n}\right)\right)(t)=\left(C_{V}^{\theta} P_{V}^{\theta} v_{n}\right)(t)+\left(L_{V}^{\theta} \Delta_{V}^{\theta} C_{V}^{\theta} x_{\theta}\left(\cdot ; 0, v_{n}\right)\right)(t), \quad \forall t \geq 0 .
$$

This implies that

$$
\left\|C_{V}^{\theta} x_{\theta}\left(\cdot ; 0, v_{n}\right)\right\|_{V\left(\mathbb{R}_{+}, Y\right)} \leq\|C\| \sup _{\theta \in \Theta}\left\|P_{V}^{\theta}\right\|\|v\|_{V\left(\mathbb{R}_{+}, X\right)}+\gamma_{V}\left\|C_{V}^{\theta} x_{\theta}\left(\cdot ; 0, v_{n}\right)\right\|_{V\left(\mathbb{R}_{+}, Y\right)} .
$$

Setting $\delta=\left\|\left|C\left\|\mid \sup _{\theta \in \Theta}\right\| P_{V}^{\theta} \|\right.\right.$ it follows that

$$
\left\|C_{V}^{\theta} x_{\theta}\left(\cdot ; 0, v_{n}\right)\right\|_{V\left(\mathbb{R}_{+}, Y\right)} \leq \frac{\delta}{1-\gamma_{V}}\|v\|_{V\left(\mathbb{R}_{+}, X\right)} .
$$

From $\left\|C_{V}^{\theta} x_{\theta}\left(\cdot ; 0, v_{n}\right)\right\|_{V\left(\mathbb{R}_{+}, Y\right)} \geq m\left\|x_{\theta}\left(\cdot ; 0, v_{n}\right)\right\|_{V\left(\mathbb{R}_{+}, X\right)}$, using $(4.25)$ we deduce that

$$
\left\|x_{\theta}\left(\cdot ; 0, v_{n}\right)\right\|_{V\left(\mathbb{R}_{+}, X\right)} \leq \frac{\delta}{m\left(1-\gamma_{V}\right)}\|v\|_{V\left(\mathbb{R}_{+}, X\right)} .
$$

Taking into account that $x_{\theta}(t ; 0, v)=x_{\theta}\left(t ; 0, v_{n}\right)$, for all $t \in[0, n)$, from (4.26) it follows that

$$
\left\|x_{\theta}(\cdot ; 0, v) \chi_{[0, n)}\right\|_{V\left(\mathbb{R}_{+}, X\right)} \leq \frac{\delta}{m\left(1-\gamma_{V}\right)}\|v\|_{V\left(\mathbb{R}_{+}, X\right)}, \quad \forall n \in \mathbb{N}^{*}
$$

Since $V \in \mathcal{U}\left(\mathbb{R}_{+}\right)$as $n \rightarrow \infty$ in (4.27), we obtain that

$$
\left\|x_{\theta}(\cdot ; 0, v)\right\|_{V\left(\mathbb{R}_{+}, X\right)} \leq \frac{\delta}{m\left(1-\gamma_{V}\right)}\|v\|_{V\left(\mathbb{R}_{+}, X\right)} .
$$

Since $\delta, m, \gamma_{V}$ do not depend on $\theta$ or $v$, we deduce that the system $(S, B \Delta C)$ is completely $\left(V\left(\mathbb{R}_{+}, X\right), V\left(\mathbb{R}_{+}, X\right)\right)$ stable. By applying Corollary 3.19 , we have that the system $(S, B \Delta C)$ is uniformly exponentially stable and the proof is complete.

The main result of this section is the following.

Theorem 4.8. The following estimation holds:

$$
r_{\text {stab }}(S, B, C) \geq \sup _{V \in \mathcal{U}\left(\mathbb{R}_{+}\right)} \frac{1}{\alpha_{V}(S, B, C)} .
$$

Proof. This follows from Theorem 4.7.

A lower bound for the stability radius of $(S)$ with respect to the perturbation structure $(B, C)$ in terms of $L^{p}$-spaces is given by the following.

Corollary 4.9. The following estimation holds:

$$
r_{\mathrm{stab}}(S, B, C) \geq \sup _{p \in[1, \infty]} \frac{1}{\alpha_{L^{p}}(S, B, C)} .
$$




\section{Applications for nonautonomous systems}

In this section, we apply the main results of this paper in order to obtain characterizations for uniform exponential stability of nonautonomous systems and deduce lower bound for the stability radius of such systems.

Let $X$ be a Banach space and let $I_{d}$ be the identity operator on $X$.

Definition 5.1. A family $\mathcal{U}=\{U(t, s)\}_{t \geq s \geq 0} \subset \boldsymbol{B}(X)$ is called an evolution family if the following properties hold:

(i) $U(t, t)=I_{d}$ and $U(t, s) U\left(s, t_{0}\right)=U\left(t, t_{0}\right)$, for all $t \geq s \geq t_{0} \geq 0$;

(ii) there are $M \geq 1$ and $\omega>0$ such that $\|U(t, s)\| \leq M e^{\omega(t-s)}$, for all $t \geq s \geq 0$;

(iii) for every $x \in X$ the mapping $(t, s) \mapsto U(t, s) x$ is continuous.

Let $\mathcal{U}=\{U(t, s)\}_{t \geq s \geq 0}$ be an evolution family on $X$. We consider the nonautonomous integral control system

$$
x_{s}\left(t ; x_{0}, u\right)=U(t, s) x_{0}+\int_{s}^{t} U(t, \tau) u(\tau) d \tau, \quad t \geq s, s \geq 0
$$

with $u \in L_{\text {loc }}^{1}\left(\mathbb{R}_{+}, X\right)$ and $x_{0} \in X$.

Definition 5.2. The system $\left(S_{\mathfrak{U}}\right)$ is said to be uniformly exponentially stable if there are $K, v>0$ such that $\left\|x_{s}\left(t ; x_{0}, 0\right)\right\| \leq K e^{-v(t-s)}\left\|x_{0}\right\|$, for all $t \geq s \geq 0$ and all $x_{0} \in X$.

Definition 5.3. Let $I, O$ be two Banach function spaces with $I, O \in \tau\left(\mathbb{R}_{+}\right)$. The system $\left(S_{\mathcal{U}}\right)$ is said to be $\left(I\left(\mathbb{R}_{+}, X\right), O\left(\mathbb{R}_{+}, X\right)\right)$ stable if the following properties hold:

(i) for every $u \in C_{0 c}\left(\mathbb{R}_{+}, X\right)$ the solution $x_{0}(\cdot ; 0, u) \in O\left(\mathbb{R}_{+}, X\right)$;

(ii) there is $\lambda>0$ such that $\left\|x_{0}(\cdot ; 0, u)\right\|_{O\left(\mathbb{R}_{+}, X\right)} \leq \lambda\|u\|_{I\left(\mathbb{R}_{+}, X\right)}$, for all $u \in C_{0 c}\left(\mathbb{R}_{+}, X\right)$.

Lemma 5.4. Let $B \in \mathcal{\tau}\left(\mathbb{R}_{+}\right), u \in B$, and $t>0$. Then, the function $\tilde{u}_{t}: \mathbb{R}_{+} \rightarrow \mathbb{R}, \tilde{u}_{t}(s)=u(s+t)$ belongs to $B$ and $\left|\tilde{u}_{t}\right|_{B} \leq|u|_{B}$.

Proof. Let

$$
v: \mathbb{R}_{+} \longrightarrow \mathbb{R}, \quad v(s)= \begin{cases}u(s), & s \geq t, \\ 0, & s \in[0, t) .\end{cases}
$$

Then $v \in B$ and $|v|_{B} \leq|u|_{B}$. Using the invariance to translations of $B$, we obtain that $\tilde{u}_{t} \in B$ and $\left|\tilde{u}_{t}\right|_{B}=|v|_{B}$, which concludes the proof.

The first main result of this section is as follows.

Theorem 5.5. Let $I, O \in \tau\left(\mathbb{R}_{+}\right)$be such that $I \in \mathcal{L}\left(\mathbb{R}_{+}\right)$and $O \in \mathcal{Q}\left(\mathbb{R}_{+}\right)$. Then, the following assertions hold:

(i) if the system $\left(S_{\mathcal{U}}\right)$ is $\left(I\left(\mathbb{R}_{+}, X\right), O\left(\mathbb{R}_{+}, X\right)\right)$ stable, then $\left(S_{\mathcal{U}}\right)$ is uniformly exponentially stable;

(ii) if $I \subset O$ and one of the spaces $I$ or $O$ belongs to $R\left(\mathbb{R}_{+}\right)$, then the system $\left(S_{u}\right)$ is uniformly exponentially stable if and only if the system $\left(S_{\mathcal{u}}\right)$ is $\left(I\left(\mathbb{R}_{+}, X\right), O\left(\mathbb{R}_{+}, X\right)\right)$ stable. 
Proof. (i) Let $\lambda>0$ be given by Definition 5.3.

Let $\Theta=\mathbb{R}_{+}$and let $\sigma: \Theta \times \mathbb{R}_{+} \rightarrow \Theta, \sigma(\theta, t)=\theta+t$. For every $(\theta, t) \in \Theta \times \mathbb{R}_{+}$, let $\Phi(\theta, t)=$ $U(t+\theta, \theta)$. Then $\pi=(\Phi, \sigma)$ is a linear skew-product flow on $X \times \Theta$. We consider the variational integral control system

$$
x_{\theta}\left(t ; x_{0}, u\right)=\Phi(\theta, t) x_{0}+\int_{0}^{t} \Phi(\sigma(\theta, s), t-s) u(s) d s, \quad t \geq 0, \theta \in \Theta .
$$

We prove that $(S)$ is $\left(I\left(\mathbb{R}_{+}, X\right), O\left(\mathbb{R}_{+}, X\right)\right)$ stable.

Indeed, let $u \in C_{0 c}\left(\mathbb{R}_{+}, X\right)$ and let $\theta \in \Theta$. We consider the function

$$
u_{\theta}: \mathbb{R}_{+} \longrightarrow X, \quad u_{\theta}(s)= \begin{cases}u(s-\theta), & s \geq \theta, \\ 0, & s \in[0, \theta) .\end{cases}
$$

Then $u_{\theta} \in C_{0 c}\left(\mathbb{R}_{+}, X\right)$ and from $I \in \tau\left(\mathbb{R}_{+}\right)$we have that $\left\|u_{\theta}\right\|_{I\left(\mathbb{R}_{+}, X\right)}=\|u\|_{I\left(\mathbb{R}_{+}, X\right)}$. From hypothesis we have that the function

$$
x_{0}\left(t ; 0, u_{\theta}\right)=\int_{0}^{t} U(t, \tau) u_{\theta}(\tau) d \tau, \quad t \geq 0
$$

belongs to $O\left(\mathbb{R}_{+}, X\right)$. Because $O\left(\mathbb{R}_{+}, X\right) \in \tau\left(\mathbb{R}_{+}\right)$, using Lemma 5.4 we have that the function

$$
\varphi: \mathbb{R}_{+} \longrightarrow X, \quad \varphi(t)=x_{0}\left(t+\theta ; 0, u_{\theta}\right)
$$

belongs to $O\left(\mathbb{R}_{+}, X\right)$ and $\|\varphi\|_{O\left(\mathbb{R}_{+}, X\right)} \leq\left\|x_{0}\left(\cdot ; 0, u_{\theta}\right)\right\|_{O\left(\mathbb{R}_{+}, X\right)}$. Observing that

$$
\begin{aligned}
\varphi(t) & =\int_{0}^{t+\theta} U(t+\theta, \tau) u_{\theta}(\tau) d \tau=\int_{\theta}^{t+\theta} U(t+\theta, \tau) u(\tau-\theta) d \tau \\
& =\int_{0}^{t} \Phi(\sigma(\theta, s), t-s) u(s) d s=x_{\theta}(t ; 0, u), \quad \forall t \geq 0,
\end{aligned}
$$

it follows that

$$
\left\|x_{\theta}(\cdot ; 0, u)\right\|_{O\left(\mathbb{R}_{+}, X\right)} \leq\left\|x_{0}\left(\cdot ; 0, u_{\theta}\right)\right\|_{O\left(\mathbb{R}_{+}, X\right)} \leq \lambda\left\|u_{\theta}\right\|_{I\left(\mathbb{R}_{+}, X\right)}=\lambda\|u\|_{I\left(\mathbb{R}_{+}, X\right)} .
$$

Since $u \in C_{0 c}\left(\mathbb{R}_{+}, X\right)$ and $\theta \in \Theta$ were arbitrary, we deduce that the system (S) is $\left(I\left(\mathbb{R}_{+}, X\right), O\left(\mathbb{R}_{+}, X\right)\right)$ stable. By applying Theorem 3.9(i) we deduce that the system $(S)$ is uniformly exponentially stable, so there are $K, v>0$ such that

$$
\left\|\Phi(\theta, t) x_{0}\right\| \leq K e^{-v t}\left\|x_{0}\right\|, \quad \forall t \geq 0, \forall x_{0} \in X .
$$

This implies that

$$
\left\|x_{s}\left(t ; x_{0}, 0\right)\right\|=\left\|U(t, s) x_{0}\right\|=\left\|\Phi(s, t-s) x_{0}\right\| \leq K e^{-v(t-s)}\left\|x_{0}\right\|, \quad \forall t \geq s \geq 0, \forall x_{0} \in X .
$$

In conclusion, the system $\left(S_{\mathcal{U}}\right)$ is uniformly exponentially stable.

(ii) Necessity follows using similar arguments as those used in the proof of Theorem 3.9. Sufficiency follows from (i). 
Remark 5.6. From Example 3.10 we deduce that the result given by Theorem 5.5 is the most general in this topic. Precisely, if $I \notin \mathcal{L}\left(\mathbb{R}_{+}\right)$or $O \notin Q\left(\mathbb{R}_{+}\right)$, then the $\left(I\left(\mathbb{R}_{+}, X\right), O\left(\mathbb{R}_{+}, X\right)\right)$ stability of the system $\left(S_{\mathcal{U}}\right)$ does not assure the uniform exponential stability of $\left(S_{\mathcal{U}}\right)$.

Definition 5.7. Let $I, O \in \tau\left(\mathbb{R}_{+}\right)$. The system $\left(S_{\mathcal{U}}\right)$ is said to be completely $\left(I\left(\mathbb{R}_{+}, X\right), O\left(\mathbb{R}_{+}, X\right)\right)$ stable if for every $u \in I\left(\mathbb{R}_{+}, X\right)$, the solution $x_{0}(\cdot ; 0, u) \in O\left(\mathbb{R}_{+}, X\right)$.

Remark 5.8. If the system $\left(S_{\mathcal{U}}\right)$ is completely $\left(I\left(\mathbb{R}_{+}, X\right), O\left(\mathbb{R}_{+}, X\right)\right)$ stable, then it makes sense to consider the linear operator

$$
p: I\left(\mathbb{R}_{+}, X\right) \longrightarrow O\left(\mathbb{R}_{+}, X\right), \quad p(u)=x_{0}(\cdot ; 0, u)
$$

It is easy to see that $D$ is closed, so it is bounded.

Remark 5.9. If the system $\left(S_{\mathcal{U}}\right)$ is completely $\left(I\left(\mathbb{R}_{+}, X\right), O\left(\mathbb{R}_{+}, X\right)\right)$ stable, then $\left(S_{\mathcal{U}}\right)$ is $\left(I\left(\mathbb{R}_{+}, X\right), O\left(\mathbb{R}_{+}, X\right)\right)$ stable (with $\left.\lambda=\|P\|\right)$.

As an application of Theorem 5.5 and using Remark 5.9 we obtain the following.

Theorem 5.10. Let $I, O \in \tau\left(\mathbb{R}_{+}\right)$be such that $I \in \mathcal{L}\left(\mathbb{R}_{+}\right)$and $O \in Q\left(\mathbb{R}_{+}\right)$. Then, the following assertions hold:

(i) if the system $\left(S_{\mathcal{U}}\right)$ is completely $\left(I\left(\mathbb{R}_{+}, X\right), O\left(\mathbb{R}_{+}, X\right)\right)$ stable, then $\left(S_{\mathcal{U}}\right)$ is uniformly exponentially stable;

(ii) if I $\subset O$ and one of the spaces $I$ or $O$ belongs to $R\left(\mathbb{R}_{+}\right)$, then the system $\left(S_{\mathcal{U}}\right)$ is uniformly exponentially stable if and only if the system $\left(S_{\mathcal{U}}\right)$ is completely $\left(I\left(\mathbb{R}_{+}, X\right), O\left(\mathbb{R}_{+}, X\right)\right)$ stable.

Corollary 5.11. If $W \in R\left(\mathbb{R}_{+}\right)$, then the system $\left(S_{\mathcal{U}}\right)$ is uniformly exponentially stable if and only if the system $\left(S_{\mathfrak{U}}\right)$ is completely $\left(W\left(\mathbb{R}_{+}, X\right), W\left(\mathbb{R}_{+}, X\right)\right)$ stable.

Corollary 5.12. Let $p, q \in[1, \infty]$ with $(p, q) \neq(1, \infty)$. The following assertions hold:

(i) if the system $\left(S_{\mathcal{U}}\right)$ is completely $\left(L^{p}\left(\mathbb{R}_{+}, X\right), L^{q}\left(\mathbb{R}_{+}, X\right)\right)$ stable, then $\left(S_{\mathcal{U}}\right)$ is uniformly exponentially stable;

(ii) if $p \leq q$, then the system $\left(S_{\mathfrak{U}}\right)$ is uniformly exponentially stable if and only if it is completely $\left(L^{p}\left(\mathbb{R}_{+}, X\right), L^{q}\left(\mathbb{R}_{+}, X\right)\right)$ stable.

Remark 5.13. For $p, q \in[1, \infty)$, Corollary 5.12 (i) was firstly proved by Datko in [16]. Recently, for $p=q \in[1, \infty)$, Corollary 5.11 was proved by Clark et al. in [11], using evolution semigroups techniques. A different proof for this corollary was given by Megan et al. in [23]. The autonomous case was treated by van Neerven in [30], for $p=q \in[1, \infty)$.

Corollary 5.14. Let $U, Y \in\left\{C_{b}\left(\mathbb{R}_{+}, \mathbb{R}\right), C_{0}\left(\mathbb{R}_{+}, \mathbb{R}\right), C_{00}\left(\mathbb{R}_{+}, \mathbb{R}\right)\right\}$ with $U \subset Y$. The system $\left(S_{\mathcal{U}}\right)$ is uniformly exponentially stable if and only if the system $\left(S_{\mathcal{U}}\right)$ is completely $\left(U\left(\mathbb{R}_{+}, X\right), Y\left(\mathbb{R}_{+}, X\right)\right)$ stable.

Proof. Necessity is immediate.

Sufficiency follows from Theorem 5.10 observing that the complete $\left(U\left(\mathbb{R}_{+}, X\right), Y\left(\mathbb{R}_{+}, X\right)\right)$ stability implies the $\left(L^{\infty}\left(\mathbb{R}_{+}, X\right), L^{\infty}\left(\mathbb{R}_{+}, X\right)\right)$ stability of $\left(S_{\mathcal{U}}\right)$. 
Remark 5.15. A distinct proof of Corollary 5.14 was given in [23]. The case $U=Y=C_{0}\left(\mathbb{R}_{+}, \mathbb{R}\right)$ was obtained in [11], reducing the nonautonomous case to the autonomous case and using evolution semigroups.

An interesting application of the results obtained in the fourth section is the study of the persistence of stability properties for the case of nonautonomous systems. We mention that the nonautonomous case was treated by Hinrichsen et al. in valuable papers (see $[17-19,41]$ ), which represent the starting point for many research studies in this area.

In what follows, we will deduce a lower bound for the stability radius of nonautonomous systems, as a consequence of the behavior in the variational case, providing the connections between these cases.

Let $X$ be a Banach space and let $\mathcal{U}=\{U(t, s)\}_{t \geq s \geq 0}$ be an evolution family on $X$.

Remark 5.16. For every $P \in \mathcal{C}_{s}\left(\mathbb{R}_{+}, B(X)\right)$ (see, e.g., [14]) there is a unique evolution family $\mathcal{U}_{P}=\left\{U_{P}(t, s)\right\}_{t \geq s \geq 0}$ such that

$$
U_{P}(t, s) x=U(t, s) x+\int_{s}^{t} U(t, \tau) P(\tau) U_{P}(\tau, s) x d \tau, \quad \forall t \geq s \geq 0, \forall x \in X .
$$

We consider the nonautonomous integral control system

$$
x_{s}\left(t ; x_{0}, u\right)=U(t, s) x_{0}+\int_{s}^{t} U(t, \tau) u(\tau) d \tau, \quad t \geq s, \quad s \geq 0
$$

with $u \in L_{\text {loc }}^{1}\left(\mathbb{R}_{+}, X\right), x_{0} \in X$, and respectively, for every $P \in \mathcal{C}_{s}\left(\mathbb{R}_{+}, B(X)\right)$ we consider the perturbed system

$$
x_{s}\left(t ; x_{0}, u\right)=U_{P}(t, s) x_{0}+\int_{s}^{t} U_{P}(t, \tau) u(\tau) d \tau, \quad t \geq s, \quad s \geq 0
$$

with $u \in L_{\text {loc }}^{1}\left(\mathbb{R}_{+}, X\right), x_{0} \in X$.

Let $U, Y$ be Banach spaces, let $B \in \mathcal{C}_{s}\left(\mathbb{R}_{+}, \mathcal{B}(U, X)\right)$ and let $C \in \mathcal{C}_{s}\left(\mathbb{R}_{+}, \mathcal{B}(X, Y)\right)$. We consider the system $\left(S_{\mathcal{U}}, B, C\right)$ described by the following integral model

$$
\begin{gathered}
x_{s}\left(t ; x_{0}, u\right)=U(t, s) x_{0}+\int_{s}^{t} U(t, \tau) B(\tau) u(\tau) d \tau, \quad t \geq s, \quad s \geq 0, \\
y_{s}\left(t ; x_{0}, u\right)=C(t) x_{s}\left(t ; x_{0}, u\right), \quad t \geq s, \quad s \geq 0,
\end{gathered}
$$

with $u \in L_{\text {loc }}^{1}\left(\mathbb{R}_{+}, U\right), x_{0} \in X$.

In what follows we suppose that $\left(S_{\mathfrak{U}}\right)$ is uniformly exponentially stable. The stability radius of $\left(S_{\mathcal{U}}\right)$ with respect to the perturbation structure $(B, C)$ is defined by

$$
\begin{aligned}
r_{\text {stab }}\left(S_{\mathcal{U}}, B, C\right)=\sup \{r & \geq 0: \forall \Delta \in \mathcal{C}_{s}\left(\mathbb{R}_{+}, B(Y, U)\right) \text { with }\|\Delta\| \mid \leq r \\
& \left.\Longrightarrow\left(S_{\mathcal{U}}, B \Delta C\right) \text { is uniformly exponentially stable }\right\} .
\end{aligned}
$$

Let $V \in \mathcal{U}\left(\mathbb{R}_{+}\right)$. Since the system $\left(S_{\mathcal{U}}\right)$ is uniformly exponentially stable, we have that $\left(S_{\mathcal{U}}\right)$ is completely $\left(V\left(\mathbb{R}_{+}, X\right), V\left(\mathbb{R}_{+}, X\right)\right)$ stable, so it makes sense to consider the linear operator

$$
p_{V}: V\left(\mathbb{R}_{+}, X\right) \rightarrow V\left(\mathbb{R}_{+}, X\right), \quad\left(p_{V} u\right)(t)=\int_{0}^{t} U(t, \tau) u(\tau) d \tau .
$$

It is easy to verify that $p_{V}$ is closed, so it is bounded. 
We consider the operators

$$
\begin{array}{ll}
B_{V}: V\left(\mathbb{R}_{+}, U\right) \longrightarrow V\left(\mathbb{R}_{+}, X\right), & \left(\mathcal{B}_{V} u\right)(t)=B(t) u(t), \\
\mathcal{C}_{V}: V\left(\mathbb{R}_{+}, U\right) \longrightarrow V\left(\mathbb{R}_{+}, X\right), & \left(\mathcal{C}_{V} u\right)(t)=C(t) u(t),
\end{array}
$$

and we note that $\boldsymbol{B}_{V}, \mathcal{C}_{V}$ are bounded linear operators with $\left\|\boldsymbol{B}_{V}\right\| \leq\|B\| \mid$ and $\left\|\mathcal{C}_{V}\right\| \leq\|C\| \mid$.

Definition 5.17. The operator

$$
\mathcal{L}_{V}: V\left(\mathbb{R}_{+}, U\right) \longrightarrow V\left(\mathbb{R}_{+}, Y\right), \quad \mathcal{L}_{V}(u)=\left(\mathcal{C}_{V} p_{V} \aleph_{V}\right)(u),
$$

is called the input-output operator associated with the system $\left(S_{\mathfrak{U}}, B, C\right)$.

In what follows, we suppose that there is $m>0$ such that $\|C(t) x\|_{Y} \geq m\|x\|$, for all $x \in X$ and all $t \geq 0$.

The second main result of this section is the following.

Theorem 5.18. The following estimation holds:

$$
r_{\text {stab }}\left(S_{\mathcal{U}}, B, C\right) \geq \frac{1}{\left\|\mathcal{L}_{V}\right\|}
$$

Proof. Let $\Delta \in \mathcal{C}_{s}\left(\mathbb{R}_{+}, \mathbb{B}(Y, U)\right)$ be such that $\left\|\left|\Delta\|\mid\| \mathcal{L}_{V} \|<1\right.\right.$.

Let $\Theta=\mathbb{R}_{+}, \sigma: \Theta \times \mathbb{R}_{+} \longrightarrow \Theta, \sigma(\theta, t)=\theta+t$ and let $\Phi(\theta, t)=U(t+\theta, \theta)$, for all $\theta, t \geq 0$. Then $\pi=(\Phi, \sigma)$ is a linear skew-product flow. We consider the variational integral control system

$$
x_{\theta}\left(t ; x_{0}, u\right)=\Phi(\theta, t) x_{0}+\int_{0}^{t} \Phi(\sigma(\theta, s), t-s) u(s) d s, \quad t \geq 0, \theta \in \Theta
$$

with $u \in L_{\text {loc }}^{1}\left(\mathbb{R}_{+}, X\right)$ and $x_{0} \in X$ and, respectively, the system $(S, B, C)$ given by

$$
\begin{gathered}
x_{\theta}\left(t, x_{0}, u\right)=\Phi(\theta, t) x_{0}+\int_{0}^{t} \Phi(\sigma(\theta, s), t-s) B(\sigma(\theta, s)) u(s) d s, \quad t \geq 0, \theta \in \Theta, \\
y_{\theta}\left(t, x_{0}, u\right)=C(\sigma(\theta, t)) x_{\theta}\left(t, x_{0}, u\right), \quad t \geq 0, \theta \in \Theta,
\end{gathered}
$$

where $x_{0} \in X$ and $u \in L_{\text {loc }}^{1}\left(\mathbb{R}_{+}, U\right)$.

For every $\theta \in \Theta$ we associate with the system $(S, B, C)$ the operators $P_{V}^{\theta}, B_{V}^{\theta}, C_{V}^{\theta}$, and $L_{V}^{\theta}$ according to the definitions in Section 4 . We denote by $\alpha_{V}(S, B, C):=\sup _{\theta \in \Theta}\left\|L_{V}^{\theta}\right\|$.

We prove that $\alpha_{V}(S, B, C) \leq\left\|\mathcal{L}_{V}\right\|$. Indeed, let $\theta \in \Theta$ and let $u \in V\left(\mathbb{R}_{+}, U\right)$. Since $V$ is invariant to translations, the function

$$
u_{\theta}: \mathbb{R}_{+} \longrightarrow U, \quad u_{\theta}(t)= \begin{cases}u(t-\theta), & t \geq \theta, \\ 0, & t \in[0, \theta),\end{cases}
$$

belongs to $V\left(\mathbb{R}_{+}, U\right)$ and $\left\|u_{\theta}\right\|_{V\left(\mathbb{R}_{+}, U\right)}=\|u\|_{V\left(\mathbb{R}_{+}, U\right)}$. In addition, using Lemma 5.4 we have that the function

$$
\varphi: \mathbb{R}_{+} \longrightarrow Y, \quad \varphi(t)=\left(\mathcal{L}_{V} u_{\theta}\right)(t+\theta)
$$


belongs to $V\left(\mathbb{R}_{+}, Y\right)$ and $\|\varphi\|_{V\left(\mathbb{R}_{+}, Y\right)} \leq\left\|\mathcal{L}_{V}\left(u_{\theta}\right)\right\|_{V\left(\mathbb{R}_{+}, Y\right)}$. We observe that

$$
\begin{aligned}
L_{V}^{\theta}(u)(t) & =C(\theta+t) \int_{0}^{t} U(\theta+t, \theta+s) B(\theta+s) u(s) d s \\
& =C(\theta+t) \int_{\theta}^{\theta+t} U(\theta+t, \tau) B(\tau) u(\tau-\theta) d \tau \\
& =C(\theta+t) \int_{0}^{\theta+t} U(\theta+t, \tau) B(\tau) u_{\theta}(\tau) d \tau \\
& =\left[\mathcal{L}_{V}\left(u_{\theta}\right)\right](t+\theta)=\varphi(t), \quad \forall t \geq 0 .
\end{aligned}
$$

This implies that

$$
\left\|L_{V}^{\theta}(u)\right\|_{V\left(\mathbb{R}_{+}, Y\right)}=\|\varphi\|_{V\left(\mathbb{R}_{+}, Y\right)} \leq\left\|\mathcal{L}_{V}\left(u_{\theta}\right)\right\|_{V\left(\mathbb{R}_{+}, Y\right)} \leq\left\|\mathcal{L}_{V}\right\|\left\|u_{\theta}\right\|_{V\left(\mathbb{R}_{+}, U\right)}=\left\|\mathcal{L}_{V}\right\|\|u\|_{V\left(\mathbb{R}_{+}, U\right)}
$$

Since $u \in V\left(\mathbb{R}_{+}, U\right)$ and $\theta \in \Theta$ were arbitrary, from the above inequality we obtain that $\left\|L_{V}^{\theta}\right\| \leq$ $\left\|\mathcal{L}_{V}\right\|$, for all $\theta \in \Theta$, so $\alpha_{V}(S, B, C) \leq\left\|\mathcal{L}_{V}\right\|$.

Since $\left\|\left|\Delta\|\mid\| \mathcal{L}_{V} \|<1\right.\right.$, we have that $\left\||\Delta \|| \alpha_{V}(S, B, C)<1\right.$. Then, from Theorem 4.7 it follows that the perturbed system $(S, B \Delta C)$ is uniformly exponentially stable, so there are $K, v>0$ such that

$$
\left\|\Phi_{B \Delta C}(\theta, t)\right\| \leq K e^{-v t}, \quad \forall t \geq 0, \forall \theta \in \Theta
$$

But

$$
\Phi_{B \Delta C}(\theta, t) x=U(t+\theta, \theta) x+\int_{0}^{t} U(\theta+t, \theta+s)(B \Delta C)(\theta+s) \Phi_{B \Delta C}(\theta, s) x d s, \quad \forall \theta, t \geq 0, \forall x \in X .
$$

which is equivalent to

$$
\Phi_{B \Delta C}(\theta, t-\theta) x=U(t, \theta) x+\int_{\theta}^{t} U(t, \tau)(B \Delta C)(\tau) \Phi_{B \Delta C}(\theta, \tau-\theta) x d \tau, \quad \forall t \geq \theta \geq 0, \forall x \in X .
$$

On the other hand, we have that

$$
U_{B \Delta C}(t, \theta) x=U(t, \theta) x+\int_{\theta}^{t} U(t, \tau)(B \Delta C)(\tau) U_{B \Delta C}(\tau, \theta) x d \tau, \quad \forall t \geq \theta \geq 0, \quad \forall x \in X .
$$

Let $\theta \geq 0$. For every $T>0$ and $x \in X$, let

$$
\varphi_{x, T}:[\theta, \theta+T] \longrightarrow X, \quad \varphi_{x, T}(\tau)=\Phi_{B \Delta C}(\theta, \tau-\theta) x-U_{B \Delta C}(\tau, \theta) x .
$$

If $M, \omega>0$ are such that $\|U(t, s)\| \leq M e^{\omega(t-s)}$, for all $t \geq s \geq 0$, then from (5.24) and (5.25) it follows that

$$
\left\|\varphi_{x, T}(t)\right\| \leq K \int_{\theta}^{t}\left\|\varphi_{x, T}(\tau)\right\| d \tau, \quad \forall t \in[\theta, \theta+T],
$$


where $K=M e^{\omega T}\|B \Delta C\|$. From (5.27), using Gronwall's lemma, we deduce that $\varphi_{x, T}(\tau)=0$, for all $\tau \in[\theta, \theta+T]$. This implies that

$$
\Phi_{B \Delta C}(\theta, \tau-\theta) x=U_{B \Delta C}(\tau, \theta) x, \quad \forall \tau \in[\theta, \theta+T], \forall x \in X .
$$

Since $T>0, x \in X$, and $\theta \in \Theta$ were arbitrary, we deduce that

$$
\Phi_{B \Delta C}(\theta, \tau-\theta)=U_{B \Delta C}(\tau, \theta), \quad \forall \tau \geq \theta \geq 0 .
$$

Then, from (5.22) we obtain that

$$
\left\|U_{B \Delta C}(\tau, \theta)\right\| \leq K e^{-v(\tau-\theta)}, \quad \forall \tau \geq \theta \geq 0 .
$$

In conclusion, the perturbed system $\left(S_{\mathfrak{U}}, B \Delta C\right)$ is uniformly exponentially stable and the proof is complete.

Concluding the above estimations, we deduce the following.

Theorem 5.19. The following estimation holds:

$$
r_{\text {stab }}\left(S_{\mathcal{U}}, B, C\right) \geq \sup _{V \in \mathcal{U}\left(\mathbb{R}_{+}\right)} \frac{1}{\left\|\mathcal{L}_{V}\right\|}
$$

A lower bound for the stability radius of nonautonomous systems in terms of $L^{p}$-spaces is given by the following.

Corollary 5.20. The following estimation holds:

$$
r_{\text {stab }}\left(S_{\mathcal{U}}, B, C\right) \geq \sup _{p \in[1, \infty]} \frac{1}{\left\|\mathcal{L}_{L^{p}}\right\|} .
$$

Remark 5.21. For the case $p \in[1, \infty)$, the result given by Corollary 5.20 was firstly proved in [19] (see [19, Theorem 3.2]) for a very general class of nonautonomous systems described by mild evolution families. A distinct proof for Corollary 5.20 was presented in [11], for $p \in[1, \infty)$, using evolution semigroups techniques (see [11, Theorem 4.2]).

Remark 5.22. A distinct approach for the estimation of the stability radius was given by Jacob in [20] for the case of time-varying systems in finite dimensional spaces. There, the author considered a time-varying system $(A, B, C)$ with $A \in L_{\text {loc }}^{\infty}\left([0, \infty), \mathbf{K}^{n \times n}\right), B \in L^{\infty}\left([0, \infty), \mathbf{K}^{n \times m}\right)$, $C \in L^{\infty}\left([0, \infty), \mathbf{K}^{q \times n}\right)$, where $\mathbf{K} \in\{\mathbb{R}, \mathbf{C}\}$. The family of input-output operators $\left\{L_{t_{0}}\right\}_{t_{0} \geq 0}$ is defined by

$$
\left(L_{t_{0}}\right) u(t):=C(t) \int_{t_{0}}^{t} \Phi_{A}(t, \tau) B(\tau) u(\tau) d \tau, \quad t \geq t_{0} \geq 0, u \in L^{p}\left(\left[t_{0}, \infty\right), \mathbf{K}^{m}\right),
$$

where $\left\{\Phi_{A}(t, s)\right\}_{t \geq s \geq 0}$ denotes the evolution family generated by A. In this case, the author proved that

$$
r_{\text {stab }}(A, B, C)=\sup _{t_{0} \geq 0} \frac{1}{\left\|L_{t_{0}}\right\|} .
$$




\section{Acknowledgments}

The author is grateful to the referee for his suggestions and comments, which led to the improvement of the paper. This work is supported by the CEEX Research Grant ET 4/2006 and by a PN II type Research Grant from West University of Timişoara.

\section{References}

[1] R. P. Agarwal, M. Bohner, and W. T. Li, Nonoscillation and Oscillation: Theory for Functional Differential Equations, vol. 267 of Monographs and Textbooks in Pure and Applied Mathematics, Marcel Dekker, New York, NY, USA, 2004.

[2] J. A. Ball, I. Gohberg, and M. A. Kaashoek, "Input-output operators of J-unitary time-varying continuous time systems," in Operator Theory in Function Spaces and Banach Lattices, vol. 75 of Operator Theory: Advances and Applications, pp. 57-94, Birkhäuser, Basel, Switzerland, 1995.

[3] V. Barbu, Mathematical Methods in Optimization of Differential Systems, vol. 310 of Mathematics and Its Applications, Kluwer Academic Publishers, Dordrecht, The Netherlands, 1994.

[4] C. Bennett and R. Sharpley, Interpolation of Operators, vol. 129 of Pure and Applied Mathematics, Academic Press, Boston, Mass, USA, 1988.

[5] M. Bohner, S. Clark, and J. Ridenhour, "Lyapunov inequalities for time scales," Journal of Inequalities and Applications, vol. 7, no. 1, pp. 61-77, 2002.

[6] M. Bohner and A. C. Peterson, Dynamic Equations on Time Scales: An Introduction with Applications, Birkhäuser, Boston, Mass, USA, 2007.

[7] C. Busse, "On the Perron-Bellman theorem for evolutionary processes with exponential growth in Banach spaces," New Zealand Journal of Mathematics, vol. 27, no. 2, pp. 183-190, 1998.

[8] C. Chicone and Y. Latushkin, Evolution Semigroups in Dynamical Systems and Differential Equations, vol. 70 of Mathematical Surveys and Monographs, American Mathematical Society, Providence, RI, USA, 1999.

[9] S.-N. Chow and H. Leiva, "Existence and roughness of the exponential dichotomy for skew-product semiflow in Banach spaces," Journal of Differential Equations, vol. 120, no. 2, pp. 429-477, 1995.

[10] S.-N. Chow and H. Leiva, "Unbounded perturbation of the exponential dichotomy and internal versus external stability in Banach spaces :an evolution semigroup approach," Journal of Differential Equations, vol. 129, pp. 509-531, 1996.

[11] S. Clark, Y. Latushkin, S. Montgomery-Smith, and T. Randolph, "Stability radius and internal versus external stability in Banach spaces: an evolution semigroup approach," SIAM Journal on Control and Optimization, vol. 38, no. 6, pp. 1757-1793, 2000.

[12] S. Clark, F. Gesztesy, and W. Renger, "Trace formulas and Borg-type theorems for matrix-valued Jacobi and Dirac finite difference operators," Journal of Differential Equations, vol. 219, no. 1, pp. 144-182, 2005.

[13] R. Curtain, "Equivalence of input-output stability and exponential stability for infinite-dimensional systems," Mathematical Systems Theory, vol. 21, no. 1, pp. 19-48, 1988.

[14] R. Curtain and H. J. Zwart, An Introduction to Infinite-Dimensional Linear Control Systems Theory, Springer, New York, NY, USA, 1995.

[15] R. Curtain and G. Weiss, "Exponential stabilization of well-posed systems by colocated feedback," SIAM Journal on Control and Optimization, vol. 45, no. 1, pp. 273-297, 2006.

[16] R. Datko, "Uniform asymptotic stability of evolutionary processes in a Banach space," SIAM Journal on Mathematical Analysis, vol. 3, pp. 428-445, 1973.

[17] D. Hinrichsen and A. J. Pritchard, "Stability radii of linear systems," Systems E Control Letters, vol. 7, no. 1, pp. 1-10, 1986.

[18] D. Hinrichsen, A. Ilchmann, and A. J. Pritchard, "Robustness of stability of time-varying linear systems," Journal of Differential Equations, vol. 82, no. 2, pp. 219-250, 1989.

[19] D. Hinrichsen and A. J. Pritchard, "Robust stability of linear evolution operators on Banach spaces," SIAM Journal on Control and Optimization, vol. 32, no. 6, pp. 1503-1541, 1994.

[20] B. Jacob, "A formula for the stability radius of time-varying systems," Journal of Differential Equations, vol. 142, no. 1, pp. 167-187, 1998. 
[21] B. Jacob and J. R. Partington, "Admissibility of control and observation operators for semigroups: a survey," in Current Trends in Operator Theory and Its Applications. Proceedings of the International Workshop on Operator Theory and Its Applications, J. A. Ball, J. W. Helton, M. Klaus, and L. Rodman, Eds., vol. 149 of Operator Theory: Advances and Applications, pp. 199-221, Birkhäuser, Basel, Switzerland, 2004.

[22] B. Jacob and J. R. Partington, "Admissible control and observation operators for Volterra integral equations," Journal of Evolution Equations, vol. 4, no. 3, pp. 333-343, 2004.

[23] M. Megan, B. Sasu, and A. L. Sasu, "Theorems of Perron type for evolution operators," Rendiconti di Matematica e delle sue Applicazioni, vol. 21, no. 1-4, pp. 231-244, 2001.

[24] M. Megan, B. Sasu, and A. L. Sasu, "On uniform exponential stability of evolution families," Rivista di Matematica della Università di Parma, vol. 4, pp. 27-43, 2001.

[25] M. Megan, A. L. Sasu, and B. Sasu, "Perron conditions and uniform exponential stability of linear skew-product semiflows on locally compact spaces," Acta Mathematica Universitatis Comenianae, vol. 70, no. 2, pp. 229-240, 2001.

[26] M. Megan, A. L. Sasu, and B. Sasu, "Stabilizability and controllability of systems associated to linear skew-product semiflows," Revista Matemática Complutense, vol. 15, no. 2, pp. 599-618, 2002.

[27] M. Megan, A. L. Sasu, and B. Sasu, "Theorems of Perron type for uniform exponential stability of linear skew-product semiflows," Dynamics of Continuous, Discrete E Impulsive Systems, vol. 12, no. 1, pp. 23-43, 2005.

[28] P. Meyer-Nieberg, Banach Lattices, Universitext, Springer, Berlin, Germany, 1991.

[29] N. van Minh, F. Räbiger, and R. Schnaubelt, "Exponential stability, exponential expansiveness, and exponential dichotomy of evolution equations on the half-line," Integral Equations and Operator Theory, vol. 32, no. 3, pp. 332-353, 1998.

[30] J. van Neerven, "Characterization of exponential stability of a semigroup of operators in terms of its action by convolution on vector-valued function spaces over $\mathbb{R}_{+}$, " Journal of Differential Equations, vol. 124, no. 2, pp. 324-342, 1996.

[31] R. J. Sacker and G. R. Sell, "Dichotomies for linear evolutionary equations in Banach spaces," Journal of Differential Equations, vol. 113, no. 1, pp. 17-67, 1994.

[32] A. L. Sasu and B. Sasu, "A lower bound for the stability radius of time-varying systems," Proceedings of the American Mathematical Society, vol. 132, no. 12, pp. 3653-3659, 2004.

[33] B. Sasu and A. L. Sasu, "Stability and stabilizability for linear systems of difference equations," Journal of Difference Equations and Applications, vol. 10, no. 12, pp. 1085-1105, 2004.

[34] A. L. Sasu, "Exponential dichotomy for evolution families on the real line," Abstract and Applied Analysis, vol. 2006, Article ID 31641, 16 pages, 2006.

[35] A. L. Sasu and B. Sasu, "Exponential dichotomy on the real line and admissibility of function spaces," Integral Equations and Operator Theory, vol. 54, no. 1, pp. 113-130, 2006.

[36] B. Sasu and A. L. Sasu, "Input-output conditions for the asymptotic behavior of linear skew-product flows and applications," Communications on Pure and Applied Analysis, vol. 5, no. 3, pp. 551-569, 2006.

[37] B. Sasu and A. L. Sasu, "Exponential trichotomy and $p$-admissibility for evolution families on the real line," Mathematische Zeitschrift, vol. 253, no. 3, pp. 515-536, 2006.

[38] B. Sasu, "New criteria for exponential expansiveness of variational difference equations," Journal of Mathematical Analysis and Applications, vol. 327, no. 1, pp. 287-297, 2007.

[39] G. Weiss, "Admissibility of unbounded control operators," SIAM Journal on Control and Optimization, vol. 27, no. 3, pp. 527-545, 1989.

[40] G. Weiss, "Transfer functions of regular linear systems. I. Characterizations of regularity," Transactions of the American Mathematical Society, vol. 342, no. 2, pp. 827-854, 1994.

[41] F. Wirth and D. Hinrichsen, "On stability radii of infinite-dimensional time-varying discrete-time systems," IMA Journal of Mathematical Control and Information, vol. 11, no. 3, pp. 253-276, 1994. 


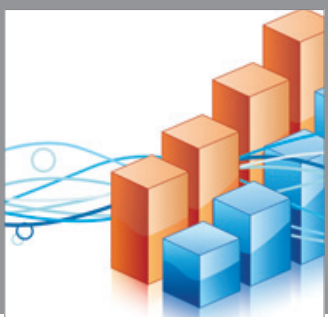

Advances in

Operations Research

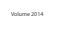

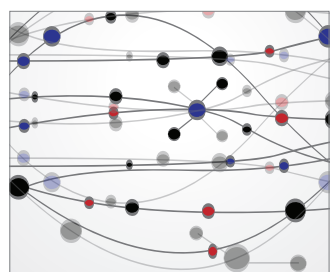

\section{The Scientific} World Journal
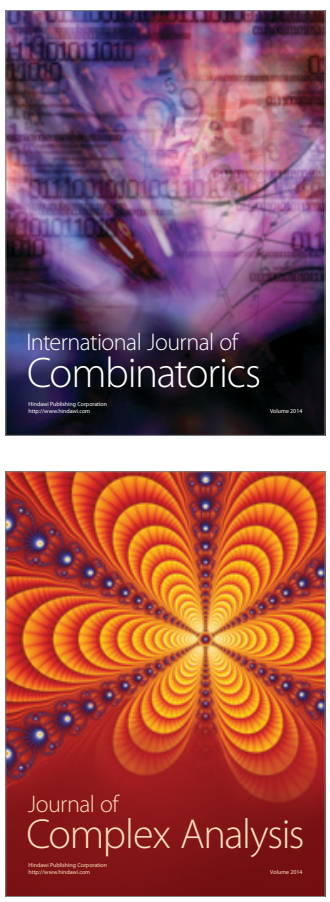

International Journal of

Mathematics and

Mathematical

Sciences
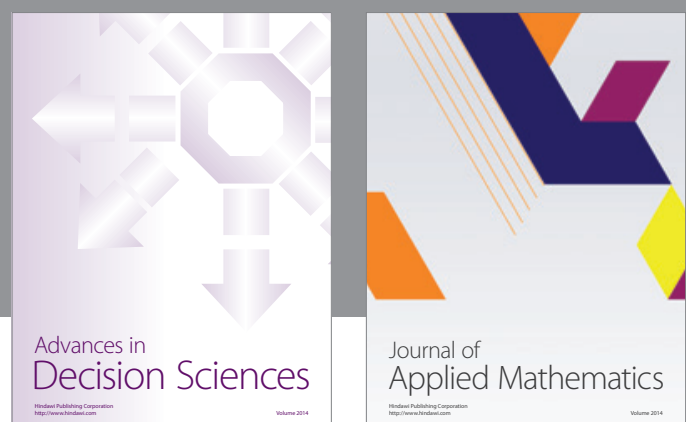

Journal of

Applied Mathematics
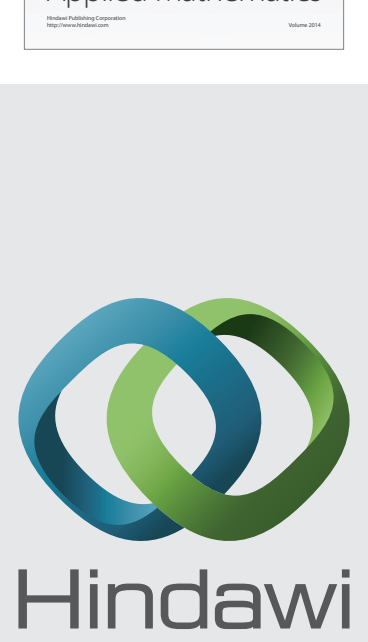

Submit your manuscripts at http://www.hindawi.com
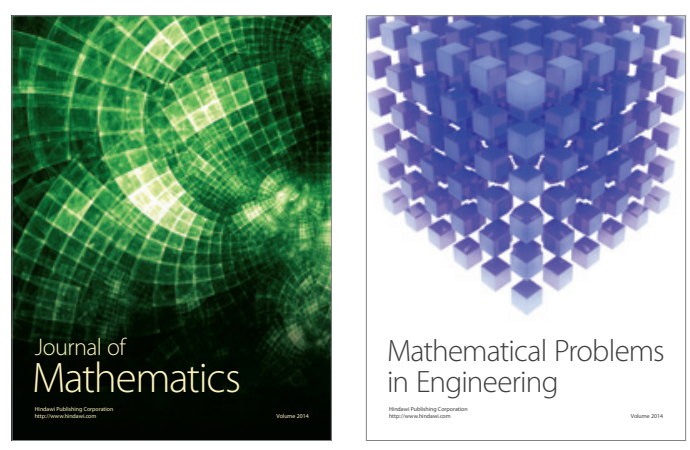

Mathematical Problems in Engineering
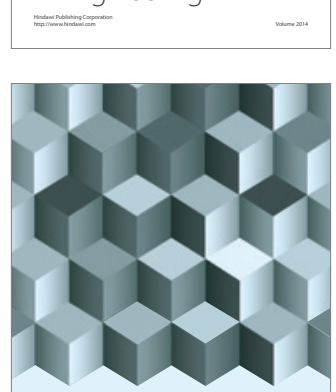

Journal of

Function Spaces
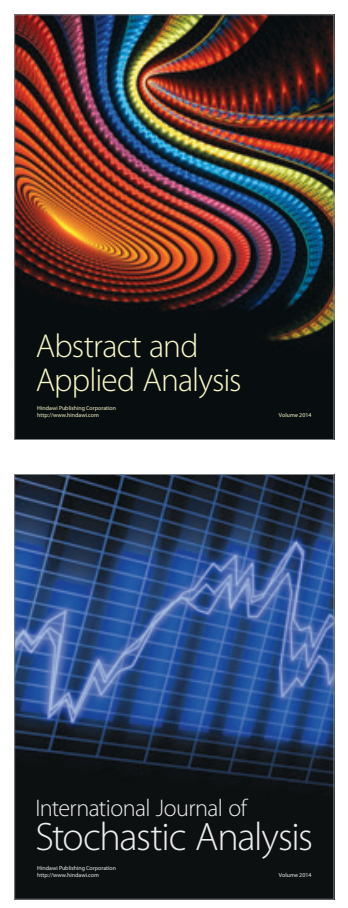

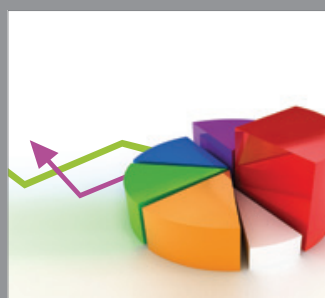

ournal of

Probability and Statistics

Promensencen
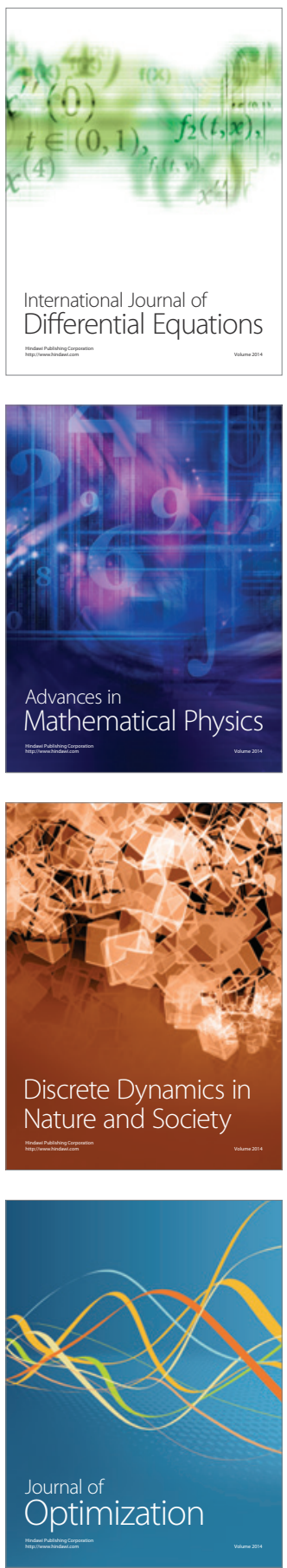